\section{UCL}

Université

catholique

de Louvain
I NSTITUT DE STATISTIQUE

B I O S T A T I S T I Q U E E T

SCIENCES ACTUARIELLES

( I S B A )
DISCUSSION PAPER

$2018 / 14$

A switching microstructure model for stock prices 


\title{
A switching microstructure model for stock prices
}

\author{
Donatien Hainaut* \\ Institute of Statistics, Bio-statistics and actuarial science (ISBA), \\ Université Catholique de Louvain, Belgium. \\ Stephane Goutte ${ }^{\dagger}$ \\ Laboratoire d'Economie Dionysien (LED), \\ Université Paris 8, France.
}

May 25, 2018

\begin{abstract}
This article proposes a microstructure model for stock prices in which parameters are modulated by a Markov chain determining the market behaviour. In this approach, called the switching microstructure model (SMM), the stock price is the result of the balance between the supply and the demand for shares. The arrivals of bid and ask orders are represented by two mutuallyand self-excited processes. The intensities of these processes converge to a mean reversion level that depends upon the regime of the Markov chain. The first part of this work studies the mathematical properties of the SMM. The second part focuses on the econometric estimation of parameters. For this purpose, we combine a particle filter with a Markov Chain Monte Carlo (MCMC) algorithm. Finally, we calibrate the SMM with two and three regimes to daily returns of the S\&P500 and compare them with a non switching model.
\end{abstract}

Keywords: Hawkes process, Switching process, microstructure

\section{Introduction}

As emphasized in the review of Bouchaud (2010), the market microstructure literature aims to explain the role of market orders on stock prices. The understanding of this relationship has significantly progressed during the last decade. For example, Bouchaud et al. (2009) explain that, because market liquidity may be low, large orders to buy or sell are only traded incrementally, over periods of time as long as weeks. As a result, order flow is a persistent long-memory process. Bacry and Muzy (2014) mention that this persistence of information causes endogeneity in stocks markets and contradicts the classical theory in which prices are driven by an exogenous flow of information.

To duplicate the endogeneity in prices, Bouchaud et al. (2010) propose a model of price fluctuations by generalizing Kyle's approach (1985) according to which the price is the result of the balance (up to a noise term) between bid and ask orders. Cont et al. (2013) study the price impact of order book events using the NYSE TAQ data for 50 U.S. stocks. They show that, over short time

\footnotetext{
*Postal address: Voie du Roman Pays 20, 1348 Louvain-la-Neuve (Belgium) . E-mail to: donatien.hainaut(at)uclouvain.be

${ }^{\dagger}$ Postal address: Université Paris 8 (LED), 2 rue de la Liberté, 93526 Saint-Denis Cedex, France E-mail to: stephane.goutte(at)univ-paris8.fr
} 
intervals, price changes are mainly driven by the imbalance between supply and demand. Kelly and Yudovina (2017) model the limit order book on short time scales, where the dynamics are driven by stochastic fluctuations between supply and demand. Horst and Paulsen (2017) study the limit properties of order books.

Bacry et al. (2013 a) reproduce the microstructure noise with multivariate Hawkes processes associated with positive and negative jumps of the asset prices. Bacry et al. (2013 b) characterise the exact macroscopic diffusion limit of this model and show in particular its ability to reproduce empirical stylised fact such as the Epps effect and the lead-lag effect. Bacry and Muzy (2014) extend this approach to prices and volumes with self and mutually excited processes. This particular category of point processes was developed by Hawkes (1971a, b) and Hawkes and Oakes (1974). In its simplest version, the intensity of jumps is persistent and suddenly increases as soon as a jump occurs in the asset price. Bowsher (2002), Hautsch (2004) and Large (2005) illustrate that Hawkes processes capture the dynamics in financial point processes remarkably well. This indicates that the cluster structure implied by the self-exciting nature of these processes provides a reasonable description of the timing structure of events in financial markets. Hardiman and Bouchaud (2014) propose a method to evaluate the integral of the Hawkes kernel, called the branching ratio which is a measure of markets endogeneity. Da Fonseca and Zaatour (2014) provide explicit formulas for moments and the autocorrelation function of the number of jumps over a given interval for the Hawkes process. Based on these moments, they propose an estimation method. Filimonov and Sornette (2015) study the pitfalls in the calibration of Hawkes processes to high frequency data. Jaisson and Rosenbaum (2015) show that nearly unstable Hawkes processes asymptotically behave like integrated Cox-Ingersoll-Ross models. Bacry and Muzy (2016) demonstrate that the jumps correlation matrix of a multivariate Hawkes process is related to the Hawkes kernel matrix by a system of Wiener-Hopf integral equations. This relation is next used to calibrate microstructure models to EuroStoxx (FSXE) and EuroBund (FGBL) future contracts. Bormetti et al. (2015) propose a Hawkes factor model to capture the time clustering of jumps and the high synchronization of jumps across assets. Hainaut (2016 a) introduces clustering of shocks in the dynamic of short term rates with Hawkes processes. Ait-Sahalia et al. (2015) use Hawkes processes to study the level of contagion between stocks markets. Chavez-Demoulin and McGill (2012) model excesses of high-frequency financial time series via a Hawkes process. Hainaut (2016 b) adapts the microstructure model of Bacry and Muzy (2014) to explain the behaviour of swap rates. Lee and Seo (2017) examines the theoretical and empirical perspectives for the symmetric Hawkes model of the price tick structure. Whereas Hainaut (2017) reveals that time changed Lévy processes with self-excited clocks explain the clustering of jumps of S\&P 500 and Eurostoxx 50 index. A detailed survey of other applications of Hawkes processes in finance is available in Bacry et al. (2015).

Given that economic cycles influence the trading behaviour and drive up or down the stocks market, we propose a microstructure model allowing for changes of trading dynamics. Our model is an extension of Bacry et al. (2013) in two directions. First, our model allows for regime shifts in the mean reversion level of orders arrivals. Second, the sizes of orders are random positive variables. In this new approach, called the "Switching Microstructure Model" (SMM), each state of the Markov chain represents a particular trading trend. Our approach is also related to the work of Wang et al. (2012) who study an univariate Markov-modulated Hawkes process, with jumps at discrete occurence times. After a complete study of SMM mathematical properties, we develop an estimation procedure that combines a particle filter with a Markov Chain Monte Carlo algorithm. We can draw a parallel between our approach and the research done at a macro-level that emphasizes the strong link between economic cycles and the dynamic of markets. For example, Guidolin and 
Timmermann (2005) present evidence of persistent 'bull' and 'bear' regimes in UK stock and bond returns. Guidolin and Timmermann (2008) obtain similar results for international stock markets. Hainaut and MacGilchrist (2012) use Markov-modulated copulas to filter economic cycles in the French stocks and bonds markets. They consider the economic implication of this relation from the perspective of an investor's portfolio allocation. Whereas Al-Anaswah and Wilfing (2011) estimate a two regimes Markov-switching specification of speculative bubbles. Recently, Branger et al. (2014) compares the correlations between asset returns induced by regime switching models with jumps and models with contagious jumps.

The paper proceeds as follows. Section 2 presents the high frequency dynamic of prices. Section 3 proposes closed form and semi-closed form expressions for moments and moment generating functions of jumps intensities and stock prices. The rest of the article focuses on the estimation of SMM parameters. Given that stock prices do not have an analytical probability distribution and that state variables are not observable, the estimation of parameters is done with a particle Markov Chain Monte Carlo algorithm. The SMM with two and three regimes is next fitted on daily data of the S\&P 500 index. Our analysis confirms that the switching microstructure market model outperforms its non-switching equivalent version. Furthermore, each regime is clearly identified to a trading trend and to a level of market stress.

\section{The switching microstructure model (SMM)}

\section{$2.1 \quad$ Stock price}

The proposed approach for the analysis of stock prices determination looks at supply and demand in the market. It finds its foundations in the economics theory. In economics, the relationship between the quantity supplied and the price is described by a curve. Under the assumption that all other economic variables are constant, quantities of supplied stocks, noted $B_{1}$, increase linearly with prices $P_{1}$. The supply curve is then described by the following relation

$$
P_{1}=L^{1}+\zeta_{1} B_{1}
$$

where $L^{1}$ and $\zeta_{1}>0$ are respectively the intercept and the elasticity of the supply curve. Under the same assumption, we can derive a demand curve that shows the relationship between the demanded quantities and prices. This demand curve has the usual downward slope, indicating that as the price increases (everything else being equal), the demanded quantity of stocks falls. The equation defining this line is the following

$$
P_{2}=L^{2}-\zeta_{2} B_{2},
$$

where $L^{2}$ and $\zeta_{2}$ are respectively the intercept and the elasticity of the demand curve. The market equilibrium occurs when the demand equals the supply, $B^{*}=B_{1}=B_{2}$, at a price $S$ such that,

$$
S=L^{2}-\zeta_{2} B^{*}=L^{1}+\zeta_{1} B^{*} .
$$

In economics, a change in market conditions is represented by a parallel shift of the demand or supply curve. Mathematically, this shift corresponds to a modification of the intercept $L^{2}$ or $L^{1}$. In order to model the dynamics of stock prices, $L^{1}$ and $L^{2}$ are then indexed by the time $t$ and are assumed to be stochastic processes. According to the relation (1), the volume of exchanged stocks at any given time is hence equal to:

$$
B_{t}^{*}=\frac{L_{t}^{2}-L_{t}^{1}}{\zeta_{1}+\zeta_{2}}
$$


and the equilibrium stocks price at time $t$ is given by

$$
S_{t}=\frac{\zeta_{1}}{\zeta_{1}+\zeta_{2}} L_{t}^{2}-\frac{\zeta_{2}}{\zeta_{1}+\zeta_{2}} L_{t}^{1}
$$

Starting from this theoretical result, we postulate that the stock price $S_{t}$ is a difference between demand $\left(L_{t}^{2}\right)$ and supply $\left(L_{t}^{1}\right)$

$$
S_{t}=\alpha_{2} L_{t}^{2}-\alpha_{1} L_{t}^{1}
$$

These processes are defined on a complete probability space $(\Omega, \mathcal{F}, P)$, with a right-continuous and complete information filtration $\mathbb{F}=\left(\mathcal{F}_{t}\right)_{t>0}$. $P$ denotes from now on the probability measure. In order to define a realistic microstructure price model while accounting for the impact of market orders as suggested by equation (4), the framework of multivariate switching Hawkes processes is well suited. Inspired from the work of Bacry et al. (2014), the supply and demand quantities that rules $S_{t}$ are related to numbers and sizes of bid-ask orders.

Let us respectively denote by $T_{1}^{1}<T_{2}^{1}<\ldots$ and $T_{1}^{2}<T_{2}^{2}<\ldots$, the sequences of arrival times of supply (bid) and demand (ask) orders. The bid order at time $T_{n}^{1}$ and the ask order at time $T_{n}^{2}$ are defined by random variables $O_{n}^{1} \in \mathcal{F}_{T_{n}^{1}}$ and $O_{n}^{2} \in \mathcal{F}_{T_{n}^{2}}$. The sequences $\left(T_{n}^{1}, O_{n}^{1}\right)$ and $\left(T_{n}^{2}, O_{n}^{2}\right)$ generate non explosive counting processes $N_{t}^{1}=\sum_{n \geq 1} 1_{\left\{T_{n}^{1} \leq t\right\}}$ and $N_{t}^{2}=\sum_{n \geq 1} 1_{\left\{T_{n}^{2} \leq t\right\}}$. From now on, $L_{t}^{1}$ and $L_{t}^{2}$ point out the processes modeling the aggregate supply and demand instead of intercepts of demand-supply curves. They are defined as the total of all bid and ask orders till time $t$ which are defined as follows:

$$
\begin{aligned}
L_{t}^{1} & =\sum_{i=1}^{N_{t}^{1}} O_{i}^{1}, \\
L_{t}^{2} & =\sum_{i=1}^{N_{t}^{2}} O_{i}^{2} .
\end{aligned}
$$

An increase of the aggregate offer of stocks causes a decline of their prices. In the opposite scenario, under the pressure of a high aggregate demand, stocks prices grow up. Then if $\alpha_{1}$ and $\alpha_{2}$ respectively denotes the permanent impact of bid and ask orders, the economics theory suggests therefore the dynamic (4) for $S_{t}$. The order sizes, $O_{i}^{1}$ and $O_{i}^{2}$, are assumed to be identically independent (i.i.d.) positive random variables: $O_{i}^{1} \sim O^{1}$ and $O_{i}^{2} \sim O^{2}$. The assumption of independence between sizes cannot be checked statistically as we do not have information about volumes. However this assumption is common in the literature about microstructure, as e.g. in Bacry et al. (2014). The densities of supply and demand orders are denoted by $\nu_{1}(z)$ and $\nu_{2}(z)$ and defined on $(0, \infty)$ sich that the moment generating function of orders, noted $\psi_{i}(\omega):=\mathbb{E}\left(e^{\omega O_{i}}\right)$ exists and is finite for $\omega \in \mathbb{C}$. First and second moments exists and are denoted $\mu_{1}=\mathbb{E}\left(O^{1}\right), \mu_{2}=\mathbb{E}\left(O^{2}\right), \eta_{1}=\mathbb{E}\left(\left(O^{1}\right)^{2}\right)$, $\eta_{2}=\mathbb{E}\left(\left(O^{2}\right)^{2}\right)$.

\subsection{Bivariate Hawkes process}

At this stage, stock prices in this model are not explicitly mean reverting. Then, there is no warranty that prices do not diverge at long term to extreme positive or negative values. However, we will see that such a divergence can be avoided by introducing dependence between arrivals of bid and ask orders. If new bid (resp. ask) orders raise the probability of ask (resp. bid) order arrivals, we 
expect a stable behaviour for $\left(S_{t}\right)_{t>0}$. Mathematically, the mutual- and self-excitation is obtained by assuming that intensities are driven by a bivariate Hawkes process but we will come back on this point later. On the other hand, flows of information and economic cycles influence the demand and supply for stocks at macro level. To introduce such a feature in our model, we assume that the economic information is carried by a hidden Markov chain with a finite number of regimes, noted $N$. The chain is a vector process $\left(\theta_{t}\right)_{t>0}$ taking values from a set of $\mathbb{R}^{N}$-valued unit vectors $E=\left\{e_{1}, \ldots, e_{N}\right\}$, where $e_{j}=(0, \ldots, 1, \ldots, 0)^{\prime}$. The filtration generated by $\left(\theta_{t}\right)_{t \geq 0}$ is denoted by $\left(\mathcal{G}_{t}\right)_{t \geq 0}$ and is a subfiltration of $\left(\mathcal{F}_{t}\right)_{t}$. The set of regimes is denoted by $\mathcal{N}:=\{1,2, \cdots, N\}$. The generator of $\theta_{t}$ is an $N \times N$ matrix $Q_{0}:=\left[q_{i, j}\right]_{i, j=1,2, \ldots, N}$ containing the instantaneous probabilities of transition. They satisfy the following standard conditions:

$$
q_{i, j} \geq 0, \quad \forall i \neq j, \quad \text { and } \quad \sum_{j=1}^{N} q_{i, j}=0, \quad \forall i \in \mathcal{N} .
$$

If $\Delta$ is a small interval of time, $q_{i, j} \Delta$ is close to the probability that the Markov chain transits from state $i$ to state $j$, with $i \neq j$. Whereas $1-q_{i, i} \Delta$ approaches the probability that the chain stays in state $i$. The matrix of transition probabilities over the time interval $[t, s]$ is denoted as $P(t, s)$ and is the matrix exponential of the generator matrix, times the length of the time interval:

$$
P(t, s)=\exp \left(Q_{0}(s-t)\right), \quad s \geq t .
$$

The elements of this matrix $, p_{i, j}(t, s), i, j \in \mathcal{N}$, defined as

$$
p_{i, j}(t, s)=P\left(\theta_{s}=e_{j} \mid \theta_{t}=e_{i}\right), \quad i, j \in \mathcal{N},
$$

are the probabilities of switching from state $i$ at time $t$ to state $j$ at time $s$. The probability of the chain being in state $i$ at time $t$, denoted by $p_{i}(t)$, depends upon the initial probabilities $p_{k}(0)$ at time $t=0$ and the transition probabilities $p_{k, i}(0, t)$, where $k=1,2, \ldots, N$, as follows:

$$
p_{i}(t)=P\left(\theta_{t}=e_{i}\right)=\sum_{k=1}^{N} p_{k}(0) p_{k, i}(0, t), \quad \forall i \in \mathcal{N} .
$$

The stationary distribution of the Markov chain is denoted $\Pi$ and is defined by the next limit

$$
\Pi=\lim _{t \rightarrow \infty} \exp \left(Q_{0} t\right)
$$

These stationary probabilities will play an important role in the calculation of the stock equilibrium price.

\subsection{Order arrival intensities}

We propose to specify the processes $N_{t}^{1}, N_{t}^{2}$, directly through their conditional arrival rates or intensities, $\left(\lambda_{t}^{1}\right)_{t \geq 0}$ and $\left(\lambda_{t}^{2}\right)_{t \geq 0}$. We assume that intensities $\lambda_{t}^{1}$ and $\lambda_{t}^{2}$ of order arrivals (OAI) are processes defined on a subfiltration $\mathcal{H}_{t} \subset \mathcal{F}_{t}$ governed by the next equations:

$$
d \lambda_{t}^{i}=\kappa_{i}\left(c_{i, t}-\lambda_{t}^{i}\right) d t+\delta_{i, 1} d L_{t}^{1}+\delta_{i, 2} d L_{t}^{2} \quad i=1,2,
$$

where $\delta_{i, j}$ for $i, j=1,2$, are constant. Coefficients $\delta_{1,2} \in \mathbb{R}^{+}$and $\delta_{2,1} \in \mathbb{R}^{+}$set the cross impact of demand on supply and vice versa. They measure the dependence between them and can capture some interesting stylized facts. E.g. if $\delta_{12}>0$, the frequency of bid orders increases when the 
demand, $L_{t}^{2}$, steps up and drives up stock prices according to equation (4). Coefficients $\delta_{1,1}$ and $\delta_{2,2}$ set the self-excitation levels. The levels of mean reversion of OAI, $c_{i, t}$ for $i=1,2$, are modulated by the Markov chain $\theta_{t}$ :

$$
c_{i, t}=c_{i}^{\top} \theta_{t}
$$

where $c_{i=1,2}$ are two strictly positive $N$-vectors: $c_{i}=\left(c_{i, 1}, \ldots, c_{i, N}\right)^{\top}$. Speeds of mean reversion of intensities, and coefficient $\left(\delta_{i, j}\right)_{i, j=1,2}$ are not modulated by the Markov chain for several reasons. This hypothesis aims to preserve the parsimony and the analytical tractability of our model. From an economic point of view, this assumption implies that the market always adjusts to new conditions with the same velocity. From a technical point of view, we will see later that modulating other parameters than $c$ makes intensities non Markov. Notice that the filtration $\mathcal{F}_{t}$ is the union of $\mathcal{H}_{t}$ and $\mathcal{G}_{t}$ augmented in the usual way by $\left\{\mathcal{V}_{t}\right\}_{t \geq 0}$. Where $\mathcal{V}_{t}$ carries complementary information about prices and aggregated order processes. Filtrations are such that $\mathcal{F}_{t}=\mathcal{G}_{t} \vee \mathcal{H}_{t} \vee \mathcal{V}_{t}$.

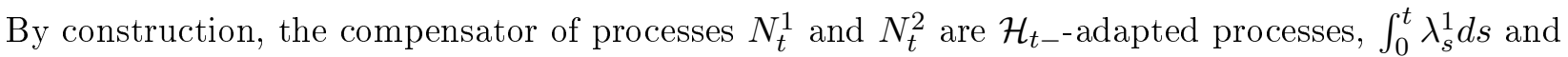
$\int_{0}^{t} \lambda_{s}^{2} d s$, such that compensated jump processes $M_{t}^{j}=N_{t}^{j}-\int_{0}^{t} \lambda_{s}^{j} d s$ are martingales. From equation (11), we infer the following lemma:

Lemma 2.1. Under the assumption that $\lambda_{t}^{1}$ and $\lambda_{t}^{2}$ are driven by the $S D E$ (11) and that $\lambda_{0}^{1}>0$, $\lambda_{0}^{2}>0$, order arrival intensities (OAI) are strictly positive processes equal to:

$$
\begin{aligned}
\lambda_{t}^{i}= & \lambda_{0}^{i}-\kappa_{i} \int_{0}^{t} e^{\kappa_{i}(s-t)}\left(\lambda_{0}^{i}-c_{i, s}\right) d s \\
& +\int_{0}^{t} \delta_{i, 1} e^{\kappa_{i}(s-t)} d L_{s}^{1}+\int_{0}^{t} \delta_{i, 2} e^{\kappa_{i}(s-t)} d L_{s}^{2} \quad i=1,2 .
\end{aligned}
$$

The proof is reported in appendix. From equation 12 we can show that $\lambda_{t}^{i}$ is related to $\lambda_{s}^{i}$ for any $s \leq t$ as follows:

$$
\begin{aligned}
\lambda_{t}^{i}= & \lambda_{s}^{i}-\kappa_{i} \int_{s}^{t} e^{\kappa_{i}(u-t)}\left(\lambda_{s}^{i}-c_{i, u}\right) d u \\
& +\int_{s}^{t} \delta_{i, 1} e^{\kappa_{i}(u-t)} d L_{u}^{1}+\int_{s}^{t} \delta_{i, 2} e^{\kappa_{i}(u-t)} d L_{u}^{2} \quad i=1,2
\end{aligned}
$$

The next section explores the properties of intensities, orders counting and cumulated orders processes. We will first show that their moments exist and demonstrate that they are Markov process.

\section{$3 \quad$ Main properties}

This section explores the mathematical features of the Switching Microstructure Model (SMM). The first subsection presents the first and second moments of orders arrival intensities (OAI). We also demonstrate in this section that processes $\lambda_{t}^{i}, L_{t}^{i}, N_{t}^{i}$ for $i=1,2$ are Markov. The second subsection studies the expected stock price and its asymptotic limit. Whereas the last subsection focuses on the probability generating and moment generating functions. 


\subsection{Moments of Order Arrival Intensities (OAI)}

The expected intensities, conditionally to the sample path of the hidden Markov chain (information carried by the augmented filtration $\mathcal{F}_{s} \vee \mathcal{G}_{t}$ with $s \leq t$ ), are provided in the following proposition. This result is next used to deduce their expectations with respect to the smaller filtration $\mathcal{F}_{s}$.

Proposition 3.1. Let us denote by $\gamma_{1}$ and $\gamma_{2}$ the following real numbers:

$$
\begin{aligned}
\gamma_{1}:= & \frac{1}{2}\left(\left(\delta_{1,1} \mu_{1}-\kappa_{1}\right)+\left(\delta_{2,2} \mu_{2}-\kappa_{2}\right)\right)+ \\
& \frac{1}{2} \sqrt{\left(\left(\delta_{1,1} \mu_{1}-\kappa_{1}\right)-\left(\delta_{2,2} \mu_{2}-\kappa_{2}\right)\right)^{2}+4 \delta_{1,2} \delta_{2,1} \mu_{1} \mu_{2}}, \\
\gamma_{2}= & \frac{1}{2}\left(\left(\delta_{1,1} \mu_{1}-\kappa_{1}\right)+\left(\delta_{2,2} \mu_{2}-\kappa_{2}\right)\right)- \\
& \frac{1}{2} \sqrt{\left(\left(\delta_{1,1} \mu_{1}-\kappa_{1}\right)-\left(\delta_{2,2} \mu_{2}-\kappa_{2}\right)\right)^{2}+4 \delta_{1,2} \delta_{2,1} \mu_{1} \mu_{2}} .
\end{aligned}
$$

Conditionally to $\mathcal{F}_{s} \vee \mathcal{G}_{t}$ with $s \leq t$, the processes $\lambda_{t}^{i}$ are Markov and their expected value of $\lambda_{t}^{i}$ is given by the next expression:

$$
\begin{aligned}
\left(\begin{array}{c}
\mathbb{E}\left(\lambda_{t}^{1} \mid \mathcal{F}_{s} \vee \mathcal{G}_{t}\right) \\
\mathbb{E}\left(\lambda_{t}^{2} \mid \mathcal{F}_{s} \vee \mathcal{G}_{t}\right)
\end{array}\right)= & V \int_{s}^{t}\left(\begin{array}{cc}
e^{\gamma_{1}(t-u)} & 0 \\
0 & e^{\gamma_{2}(t-u)}
\end{array}\right) V^{-1}\left(\begin{array}{c}
\kappa_{1} c_{1, u} \\
\kappa_{2} c_{2, u}
\end{array}\right) d u \\
& +V\left(\begin{array}{cc}
e^{\gamma_{1}(t-s)} & 0 \\
0 & e^{\gamma_{2}(t-s)}
\end{array}\right) V^{-1}\left(\begin{array}{c}
\lambda_{s}^{1} \\
\lambda_{s}^{2}
\end{array}\right),
\end{aligned}
$$

where $V, V^{-1}$ are matrices given by:

$$
\begin{gathered}
V=\left(\begin{array}{cc}
-\delta_{1,2} \mu_{2} & -\delta_{1,2} \mu_{2} \\
\left(\delta_{1,1} \mu_{1}-\kappa_{1}\right)-\gamma_{1} & \left(\delta_{1,1} \mu_{1}-\kappa_{1}\right)-\gamma_{2}
\end{array}\right), \\
V^{-1}=\frac{1}{\Upsilon}\left(\begin{array}{cc}
\left(\delta_{1,1} \mu_{1}-\kappa_{1}\right)-\gamma_{2} & \delta_{1,2} \mu_{2} \\
\gamma_{1}-\left(\delta_{1,1} \mu_{1}-\kappa_{1}\right) & -\delta_{1,2} \mu_{2}
\end{array}\right),
\end{gathered}
$$

and $\Upsilon \in \mathbb{R}$ is the determinant of $V$ defined by

$$
\Upsilon:=-\delta_{1,2} \mu_{2} \sqrt{\left(\left(\delta_{1,1} \mu_{1}-\kappa_{1}\right)-\left(\delta_{2,2} \mu_{2}-\kappa_{2}\right)\right)^{2}+4 \delta_{1,2} \delta_{2,1} \mu_{1} \mu_{2}} .
$$

Here, I points out the identity matrix of size $N \times N$.

The proof is in appendix. Knowing the expectation of $\lambda_{t}^{i}$ conditionally to the sample path of $\theta_{t}$, we can infer the unconditional expectations of intensities and prove that $\lambda_{t}^{i}$ are Markov as stated in the following proposition.

Proposition 3.2. $\lambda_{t}^{1}$ and $\lambda_{t}^{2}$ are Markov processes and their expected values conditionally to $\mathcal{F}_{s}$ for $s \leq t$, are given by the next expression:

$$
\left(\begin{array}{l}
\mathbb{E}\left(\lambda_{t}^{1} \mid \mathcal{F}_{s}\right) \\
\mathbb{E}\left(\lambda_{t}^{2} \mid \mathcal{F}_{s}\right)
\end{array}\right)=V\left(\begin{array}{c}
m_{1}\left(t, \theta_{s}\right) \\
m_{2}\left(t, \theta_{s}\right)
\end{array}\right)+V\left(\begin{array}{cc}
e^{\gamma_{1}(t-s)} & 0 \\
0 & e^{\gamma_{2}(t-s)}
\end{array}\right) V^{-1}\left(\begin{array}{c}
\lambda_{s}^{1} \\
\lambda_{s}^{2}
\end{array}\right)
$$


where $m_{1}\left(t, \theta_{s}\right)$ and $m_{2}\left(t, \theta_{s}\right)$ are respectively equal to

$$
\begin{aligned}
& m_{1}\left(t, \theta_{s}\right)=e_{1}^{\top} M_{1}\left(t, \theta_{s}\right)\left(\begin{array}{c}
\frac{1}{\Upsilon} \kappa_{1}\left(\left(\delta_{1,1} \mu_{1}-\kappa_{1}\right)-\gamma_{2}\right) \\
\frac{1}{\Upsilon} \kappa_{2} \delta_{1,2} \mu_{2}
\end{array}\right), \\
& m_{2}\left(t, \theta_{s}\right)=e_{2}^{\top} M_{1}\left(t, \theta_{s}\right)\left(\begin{array}{c}
\frac{1}{\Upsilon} \kappa_{1}\left(\gamma_{1}-\left(\delta_{1,1} \mu_{1}-\kappa_{1}\right)\right) \\
-\frac{1}{\Upsilon} \kappa_{2} \delta_{1,2} \mu_{2}
\end{array}\right),
\end{aligned}
$$

and $M_{1}\left(t, \theta_{s}\right)$ is the following time and state dependent matrix:

$$
\begin{aligned}
& M_{1}\left(t, \theta_{s}\right):=\left(\begin{array}{cc}
\theta_{s}^{\top}\left(Q_{0}-\gamma_{1} I\right)^{-1} & 0 \\
0 & \theta_{s}^{\top}\left(Q_{0}-\gamma_{2} I\right)^{-1}
\end{array}\right) \\
& \times\left(\begin{array}{cc}
{\left[\exp \left(Q_{0}(t-s)\right)-\exp \left(I \gamma_{1}(t-s)\right)\right]} & 0 \\
0 & {\left[\exp \left(Q_{0}(t-s)\right)-\exp \left(I \gamma_{2}(t-s)\right)\right]}
\end{array}\right)\left(\begin{array}{ll}
c_{1} & c_{2} \\
c_{1} & c_{2}
\end{array}\right) .
\end{aligned}
$$

See appendix for the proof. Notice that we cannot find moments and prove the Markov feature of intensities when the speed reversion or mutual excitation parameters are modulated by $\theta_{t}$. To understand this point, let us assume that $\kappa_{1}$ and $\kappa_{2}$ depends on $\theta_{t}$. In this case, the matrix $V$ and parameters $\gamma_{1}, \gamma_{2}$ involved in conditional expectations of intensities with respect to $\mathcal{F}_{s} \vee \mathcal{G}_{s}$ (proposition 3.1 are modulated by $\theta_{t}$. Calculating the expectation with respect to the filtration $\mathcal{F}_{s}$, as done in the proof of proposition 3.2 is in this case no more possible. Mainly because this requires to calculate the expectation of an integral of a product of terms related to $V, V^{-1}, \gamma_{1}, \gamma_{2}, c_{1, t}$ and $c_{2, t}$ that all are modulated by $\theta_{t}$. If we want to preserve the Markov feature of our model, the drift is the only modulable parameter. Wang et al. (2012) draw the same conclusion for an univariate switching Hawkes process.

From this last proposition, we infer the conditions that ensure the stability of the process. The OAI's remain finite $\left(\lambda_{t}^{1}<\infty\right.$ and $\lambda_{t}^{2}<\infty$ almost surely $\forall t \geq 0$ ) if only $\gamma_{1}$ and $\gamma_{2}$ are negative. In the opposite case, the limits of $\lambda_{t}^{i}$ for $i=1,2$ when $t \rightarrow \infty$ diverge to $+\infty$. If $\gamma_{1}<0$ and $\gamma_{2}<0$, the expected intensities converge toward:

$$
\lim _{t \rightarrow \infty}\left(\begin{array}{l}
\mathbb{E}\left(\lambda_{t}^{1} \mid \mathcal{F}_{s}\right) \\
\mathbb{E}\left(\lambda_{t}^{2} \mid \mathcal{F}_{s}\right)
\end{array}\right)=V\left(\begin{array}{c}
m_{1}(\infty) \\
m_{2}(\infty)
\end{array}\right)
$$

where the constant $m_{1}(\infty)$ and $m_{2}(\infty)$ are the limits of functions $m_{2}\left(t, \theta_{s}\right)$ and $m_{2}\left(t, \theta_{s}\right)$ :

$$
\begin{aligned}
m_{1}(\infty):=\lim _{t \rightarrow \infty} m_{1}\left(t, \theta_{s}\right)=\frac{1}{\Upsilon} & {\left[\kappa_{1}\left(\left(\delta_{1,1} \mu_{1}-\kappa_{1}\right)-\gamma_{2}\right) \theta_{s}^{\top}\left(Q_{0}-\gamma_{1} I\right)^{-1} \Pi c_{1}\right.} \\
& \left.+\kappa_{2} \delta_{1,2} \mu_{2} \theta_{s}^{\top}\left(Q_{0}-\gamma_{1} I\right)^{-1} \Pi c_{2}\right], \\
m_{2}(\infty):=\lim _{t \rightarrow \infty} m_{2}\left(t, \theta_{s}\right)=\frac{1}{\Upsilon} & {\left[\kappa_{1}\left(\gamma_{1}-\left(\delta_{1,1} \mu_{1}-\kappa_{1}\right)\right) \kappa_{1} \theta_{s}^{\top}\left(Q_{0}-\gamma_{2} I\right)^{-1} \Pi c_{1}\right.} \\
& \left.-\kappa_{2} \delta_{1,2} \mu_{2} \theta_{s}^{\top}\left(Q_{0}-\gamma_{2} I\right)^{-1} \Pi c_{2}\right],
\end{aligned}
$$

and $\Pi=\lim _{t \rightarrow \infty} \exp \left(Q_{0} t\right)$ is the stationary distribution of $\theta_{t}$. From propositions 15 and 3.2 , we prove in appendix that counting and cumulated orders processes are Markov ones:

Corollary 3.3. The processes $\left(N_{t}^{1}\right)_{t \geq 0},\left(N_{t}^{2}\right)_{t \geq 0},\left(L_{t}^{1}\right)_{t \geq 0}$ and $\left(L_{t}^{2}\right)_{t \geq 0}$ are Markov processes with respect to the filtration $\mathcal{F}$. 
The expectations of $L_{t}^{1}$ and $L_{t}^{2}$ admit closed form expressions that are developed in section 3.2. Let us denote $J_{t}^{i}=\left(L_{t}^{i}, N_{t}^{i}\right)$. Propositions 3.2 and 3.3 suggest that the multivariate process $\left(\lambda_{t}^{1}, J_{t}^{1}, \lambda_{t}^{2}, J_{t}^{2}, \theta_{t}\right)$ is a Markov process in the state space

$$
D=\left(\mathbb{R}^{+} \times \mathbb{R}^{+} \times \mathbb{N}\right)^{2} \times E
$$

All processes are Markov ones, adapted to $\mathcal{F}$ with càdlag paths. By construction, they are decomposable and then semi-martingales. Using the Itô's formula for semi-martingales (see e.g. Protter 2004 , theorem 32, p79), allows us to find the infinitesimal generator for any function $g: D \rightarrow \mathbb{R}$ with continuous partial derivatives $g_{\lambda_{1}}, g_{\lambda_{2}}$. If $\theta_{t}=e_{i}$, the generator of this function, denoted $\mathcal{A} g($.$) , is given by the following expression:$

$$
\begin{aligned}
\mathcal{A} g( & \left.\lambda_{t}^{1}, J_{t}^{1}, \lambda_{t}^{2}, J_{t}^{2}, e_{i}\right)=\kappa_{1}\left(c_{1, t}-\lambda_{t}^{1}\right) g_{\lambda^{1}}+\kappa_{2}\left(c_{2, t}-\lambda_{t}^{2}\right) g_{\lambda^{2}} \\
& +\lambda_{t}^{1} \int_{-\infty}^{+\infty} g\left(\lambda_{t}^{1}+\delta_{1,1} z, J_{t}^{1}+(z, 1)^{\top}, \lambda_{t}^{2}+\delta_{2,1} z, J_{t}^{2}, e_{i}\right)-g\left(\lambda_{t}^{1}, J_{t}^{1}, \lambda_{t}^{2}, J_{t}^{2}, e_{i}\right) \nu_{1}(d z) \\
& +\lambda_{t}^{2} \int_{-\infty}^{+\infty} g\left(\lambda_{t}^{1}+\delta_{1,2} z, J_{t}^{1}, \lambda_{t}^{2}+\delta_{2,2} z, J_{t}^{2}+(z, 1)^{\top}, e_{i}\right)-g\left(\lambda_{t}^{1}, J_{t}^{1}, \lambda_{t}^{2}, J_{t}^{2}, e_{i}\right) \nu_{2}(d z) \\
& +\sum_{j \neq i}^{N} q_{i, j}\left(g\left(\lambda_{t}^{1}, J_{t}^{1}, \lambda_{t}^{2}, J_{t}^{2}, e_{j}\right)-g\left(\lambda_{t}^{1}, J_{t}^{1}, \lambda_{t}^{2}, J_{t}^{2}, e_{i}\right)\right) .
\end{aligned}
$$

Under mild conditions, the expectation of $g($.$) is equal to the integral of the expected infinitesimal$ generator. Using the Fubini's theorem leads to the following result:

$$
\begin{aligned}
& \mathbb{E}\left(g\left(\lambda_{T}^{1}, J_{T}^{1}, \lambda_{T}^{2}, J_{T}^{2}, \theta_{T}\right) \mid \mathcal{F}_{t}\right) \\
& \quad=g\left(\lambda_{t}^{1}, J_{t}^{1}, \lambda_{t}^{2}, J_{t}^{2}\right)+\int_{t}^{T} \mathbb{E}\left(\mathcal{A} g\left(\lambda_{s}^{1}, J_{s}^{1}, \lambda_{s}^{2}, J_{s}^{2}, \theta_{s}\right) \mid \mathcal{F}_{t}\right) d s .
\end{aligned}
$$

The derivative of this expectation with respect to time is equal to its expected infinitesimal generator:

$$
\frac{\partial}{\partial T} \mathbb{E}\left(g\left(\lambda_{T}^{1}, J_{T}^{1}, \lambda_{T}^{2}, J_{T}^{2},, \theta_{T}\right) \mid \mathcal{F}_{t}\right)=\mathbb{E}\left(\mathcal{A} g\left(\lambda_{T}^{1}, J_{T}^{1}, \lambda_{T}^{2}, J_{T}^{2}, \theta_{T}\right) \mid \mathcal{F}_{t}\right) .
$$

We use this result later in the proof of the last proposition of this section. The remainder of this paragraph is devoted to the calculation of the variance of intensities $\lambda_{t}^{1}$ and $\lambda_{t}^{2}$. Unfortunately, the variances of these OAI do not admit any closed form expression. But the second order moment of $\lambda_{t}^{i}$ can be calculated numerically by solving a system of ordinary differential equations (ODE). Writing this system requires additional intermediate results about the expectations of mean reversion levels and OAI. The next proposition (proven in appendix) presents these expectations when the sample path of $\theta_{t}$ is observed from 0 up to time $t$.

Proposition 3.4. The expected value of $c_{j, t} \lambda_{t}^{i}$ for $i, j=1,2$ with respect to the augmented filtration 
$\mathcal{F}_{0} \vee \mathcal{G}_{t}$ is given by

$$
\begin{array}{r}
\left(\begin{array}{c}
\mathbb{E}\left(c_{1, t} \lambda_{t}^{1} \mid \mathcal{F}_{0} \vee \mathcal{G}_{t}\right) \\
\mathbb{E}\left(c_{2, t} \lambda_{t}^{1} \mid \mathcal{F}_{0} \vee \mathcal{G}_{t}\right) \\
\mathbb{E}\left(c_{1, t} \lambda_{t}^{2} \mid \mathcal{F}_{0} \vee \mathcal{G}_{t}\right) \\
\mathbb{E}\left(c_{2, t} \lambda_{t}^{2} \mid \mathcal{F}_{0} \vee \mathcal{G}_{t}\right)
\end{array}\right)=\int_{0}^{t} W \exp (F s) W^{-1} K\left(\begin{array}{c}
c_{1, s}^{2} \\
c_{2, s}^{2} \\
c_{1, s} c_{2, s}
\end{array}\right) d s \\
+W \exp (F t) W^{-1}\left(\begin{array}{c}
c_{1,0} \lambda_{0}^{1} \\
c_{2,0} \lambda_{0}^{1} \\
c_{1,0} \lambda_{0}^{2} \\
c_{2,0} \lambda_{0}^{2}
\end{array}\right),
\end{array}
$$

where $W$ is a $4 \times 4$ matrix

$$
W=\left(\begin{array}{cccc}
-\delta_{1,2} \mu_{2} & -\delta_{1,2} \mu_{2} & 0 & 0 \\
0 & 0 & -\delta_{1,2} \mu_{2} & -\delta_{1,2} \mu_{2} \\
\left(\delta_{1,1} \mu_{1}-\kappa_{1}\right)-\gamma_{1} & \left(\delta_{1,1} \mu_{1}-\kappa_{1}\right)-\gamma_{2} & 0 & 0 \\
0 & 0 & \left(\delta_{1,1} \mu_{1}-\kappa_{1}\right)-\gamma_{1} & \left(\delta_{1,1} \mu_{1}-\kappa_{1}\right)-\gamma_{2}
\end{array}\right),
$$

that admits the following inverse

$$
W^{-1}=\frac{1}{\Upsilon}\left(\begin{array}{cccc}
\left(\delta_{1,1} \mu_{1}-\kappa_{1}\right)-\gamma_{2} & 0 & \delta_{1,2} \mu_{2} & 0 \\
\gamma_{1}-\left(\delta_{1,1} \mu_{1}-\kappa_{1}\right) & 0 & -\delta_{1,2} \mu_{2} & 0 \\
0 & \left(\delta_{1,1} \mu_{1}-\kappa_{1}\right)-\gamma_{2} & 0 & \delta_{1,2} \mu_{2} \\
0 & \gamma_{1}-\left(\delta_{1,1} \mu_{1}-\kappa_{1}\right) & 0 & -\delta_{1,2} \mu_{2}
\end{array}\right)
$$

$\Upsilon$ is still defined by equation (18) whereas $F$ and $K$ are the following matrix

$$
F=\left(\begin{array}{cccc}
\gamma_{1} & 0 & 0 & 0 \\
0 & \gamma_{2} & 0 & 0 \\
0 & 0 & \gamma_{1} & 0 \\
0 & 0 & 0 & \gamma_{2}
\end{array}\right) \quad K=\left(\begin{array}{ccc}
\kappa_{1} & 0 & 0 \\
0 & 0 & \kappa_{1} \\
0 & 0 & \kappa_{2} \\
0 & \kappa_{2} & 0
\end{array}\right)
$$

$\gamma_{1}$ and $\gamma_{2}$ are defined by equations (14).

Using similar arguments to these used in the proof of proposition 3.2 we infer the unconditional expectations of the product of mean reversion levels and of intensities:

Proposition 3.5. Let us denote $\bar{c}_{1}^{2}=\left(c_{1,1}^{2}, \ldots, c_{1, N}^{2}\right), \bar{c}_{2}^{2}=\left(c_{2,1}^{2}, \ldots, c_{2, N}^{2}\right)$ and

$$
\bar{c}_{1 \times 2}=\left(c_{1,1} \times c_{2,1}, \ldots, c_{1, N} \times c_{2, N}\right) .
$$

Expectations of $c_{j, t} \lambda_{t}^{i}$ for $i, j=1,2$ with respect to the $\mathcal{F}_{0}$ are equal to

$$
\left(\begin{array}{l}
\mathbb{E}\left(c_{1, t} \lambda_{t}^{1} \mid \mathcal{F}_{0}\right) \\
\mathbb{E}\left(c_{2, t} \lambda_{t}^{1} \mid \mathcal{F}_{0}\right) \\
\mathbb{E}\left(c_{1, t} \lambda_{t}^{2} \mid \mathcal{F}_{0}\right) \\
\mathbb{E}\left(c_{2, t} \lambda_{t}^{2} \mid \mathcal{F}_{0}\right)
\end{array}\right)=W\left(X\left(t, \theta_{0}\right)+Y\left(t, \theta_{0}\right)\right)+W \exp (F t) W^{-1}\left(\begin{array}{c}
c_{1,0} \lambda_{0}^{1} \\
c_{2,0} \lambda_{0}^{1} \\
c_{1,0} \lambda_{0}^{2} \\
c_{2,0} \lambda_{0}^{2}
\end{array}\right)
$$


where $X\left(t, \theta_{0}\right)$ and $Y\left(t, \theta_{0}\right)$ are the next time-dependent vectors of dimension 4 :

$$
\begin{gathered}
X\left(t, \theta_{0}\right)=\frac{1}{\Upsilon}\left(\begin{array}{c}
\kappa_{1}\left(\left(\delta_{1,1} \mu_{1}-\kappa_{1}\right)-\gamma_{2}\right)\left(\theta_{0}\left(\frac{\left(e^{\left(Q_{0}+\gamma_{1} I\right) t}-I\right)}{Q_{0}+\gamma_{1} I}\right) c_{1}^{2}\right) \\
\kappa_{1}\left(\gamma_{1}-\left(\delta_{1,1} \mu_{1}-\kappa_{1}\right)\right)\left(\theta_{0}\left(\frac{\left(e^{\left(Q_{0}+\gamma_{2} I\right) t}-I\right)}{Q_{0}+\gamma_{2} I}\right) c_{1}^{2}\right) \\
\kappa_{1}\left(\left(\delta_{1,1} \mu_{1}-\kappa_{1}\right)-\gamma_{2}\right)\left(\theta_{0}\left(\frac{\left(e^{\left(Q_{0}+\gamma_{1} I\right) t}-I\right)}{Q_{0}+\gamma_{1} I}\right) \bar{c}_{1,2}\right) \\
\kappa_{1}\left(\gamma_{1}-\left(\delta_{1,1} \mu_{1}-\kappa_{1}\right)\right)\left(\theta_{0}\left(\frac{\left(e^{\left(Q_{0}+\gamma_{2} I\right) t}-I\right)}{Q_{0}+\gamma_{2} I}\right) \bar{c}_{1,2}\right)
\end{array}\right), \\
Y\left(t, \theta_{0}\right)=\frac{1}{\Upsilon}\left(\begin{array}{c}
-\delta_{1,2} \mu_{2} \kappa_{2}\left(\theta_{0}\left(\frac{\left(e^{\left(Q_{0}+\gamma_{1} I\right) t}-I\right)}{Q_{0}+\gamma_{1} I}\right) \bar{c}_{1,2}\right) \\
\delta_{1,2} \mu_{2} \kappa_{2}\left(\theta_{0}\left(\frac{\left(e^{\left(Q_{0}+\gamma_{1} I\right) t}-I\right)}{Q_{0}+\gamma_{1} I}\right) \bar{c}_{2}^{2}\right) \\
-\delta_{1,2} \mu_{2} \kappa_{2}\left(\theta_{0}\left(\frac{\left(e^{\left(Q_{0}+\gamma_{2} I\right) t}-I\right)}{\left.Q_{0}+\gamma_{2} I\right) t}\right) \bar{c}_{2}^{2}\right)
\end{array}\right) .
\end{gathered}
$$

As announced earlier, the last result of this subsection presents the ODE's satisfied by the second order moments of intensities. Solving them numerically allows us to evaluate the standard deviation and correlation of intensities.

Proposition 3.6. The second order moments of $\lambda_{t}$ are solution of a system of ODE:

$$
\begin{aligned}
& \left(\begin{array}{c}
\frac{\partial}{\partial t} \mathbb{E}\left(\left(\lambda_{t}^{1}\right)^{2} \mid \mathcal{F}_{0}\right) \\
\frac{\partial}{\partial t} \mathbb{E}\left(\left(\lambda_{t}^{2}\right)^{2} \mid \mathcal{F}_{0}\right) \\
\frac{\partial}{\partial t} \mathbb{E}\left(\lambda_{t}^{1} \lambda_{t}^{2} \mid \mathcal{F}_{0}\right)
\end{array}\right)=\left(\begin{array}{cccc}
2 \kappa_{1} & 0 & 0 & 0 \\
0 & 0 & 0 & 2 \kappa_{2} \\
0 & \kappa_{2} & \kappa_{1} & 0
\end{array}\right)\left(\begin{array}{l}
\mathbb{E}\left(c_{1, t} \lambda_{t}^{1} \mid \mathcal{F}_{0}\right) \\
\mathbb{E}\left(c_{2, t} \lambda_{t}^{1} \mid \mathcal{F}_{0}\right) \\
\mathbb{E}\left(c_{1, t} \lambda_{t}^{2} \mid \mathcal{F}_{0}\right) \\
\mathbb{E}\left(c_{2, t} \lambda_{t}^{2} \mid \mathcal{F}_{0}\right)
\end{array}\right)+ \\
& \left(\begin{array}{cc}
\delta_{1,1}^{2} \eta_{1} & \delta_{1,2}^{2} \eta_{2} \\
\delta_{2,1}^{2} \eta_{1} & \delta_{2,2}^{2} \eta_{2} \\
\delta_{1,1} \delta_{2,1} \eta_{1} & \delta_{1,2} \delta_{2,2} \eta_{2}
\end{array}\right)\left(\begin{array}{c}
\mathbb{E}\left(\lambda_{t}^{1} \mid \mathcal{F}_{0}\right) \\
\mathbb{E}\left(\lambda_{t}^{2} \mid \mathcal{F}_{0}\right)
\end{array}\right)+ \\
& \left(\begin{array}{ccc}
2\left(\delta_{1,1} \mu_{1}-\kappa_{1}\right) & 0 & 2 \delta_{1,2} \mu_{2} \\
0 & 2\left(\delta_{2,2} \mu_{2}-\kappa_{2}\right) & 2 \delta_{2,1} \mu_{1} \\
\delta_{2,1} \mu_{1} & \delta_{1,2} \mu_{2} & \left(\begin{array}{c}
\delta_{1,1} \mu_{1}-\kappa_{1} \\
+\delta_{2,2} \mu_{2}-\kappa_{2}
\end{array}\right)
\end{array}\right)\left(\begin{array}{c}
\mathbb{E}\left(\left(\lambda_{t}^{1}\right)^{2} \mid \mathcal{F}_{0}\right) \\
\mathbb{E}\left(\left(\lambda_{t}^{2}\right)^{2} \mid \mathcal{F}_{0}\right) \\
\mathbb{E}\left(\lambda_{t}^{1} \lambda_{t}^{2} \mid \mathcal{F}_{0}\right)
\end{array}\right)
\end{aligned}
$$

with the initial conditions $\mathbb{E}\left(\left(\lambda_{0}^{1}\right)^{2} \mid \mathcal{F}_{0}\right)=\left(\lambda_{0}^{1}\right)^{2}, \mathbb{E}\left(\left(\lambda_{0}^{2}\right)^{2} \mid \mathcal{F}_{0}\right)=\left(\lambda_{0}^{2}\right)^{2}$ and $\mathbb{E}\left(\lambda_{t}^{1} \lambda_{t}^{2} \mid \mathcal{F}_{0}\right)=\lambda_{0}^{1} \lambda_{0}^{2}$.

We refer the reader to the appendix for the proof of this result, which is based on relation 26. The next subsection studies the equilibrium price of stocks, such as defined by equation (4).

\subsection{Stock price}

This subsection presents the expectation, the asymptotic limit and the momeng generating function of stock prices. Remember that in the SMM, the price is determined by the equilibrium between 
the aggregate supply and offer as follows:

$$
S_{t}=\alpha_{2} \int_{0}^{t} d L_{s}^{2}-\alpha_{1} \int_{0}^{t} d L_{s}^{1} .
$$

Given that intensities at time $t^{-}$are independent from jumps at time $t$, then the expected stock price is equal to

$$
\mathbb{E}\left(S_{t} \mid \mathcal{F}_{0}\right)=\alpha_{2} \mu_{2} \int_{0}^{t} \mathbb{E}\left(\lambda_{s}^{2} \mid \mathcal{F}_{0}\right) d s-\alpha_{1} \mu_{1} \int_{0}^{t} \mathbb{E}\left(\lambda_{s}^{1} \mid \mathcal{F}_{0}\right)
$$

If we insert the expressions 3.2 of the conditionial expectations, we prove by direct integration that the expected price, as stated in the following proposition.

Proposition 3.7. The expected stock price in the SMM is equal to:

$$
\begin{aligned}
\mathbb{E}\left(S_{t} \mid \mathcal{F}_{0}\right) & =S_{0}+\left(\begin{array}{c}
-\alpha_{1} \mu_{1} \\
\alpha_{2} \mu_{2}
\end{array}\right)^{\top} V\left(\begin{array}{c}
\int_{0}^{t} m_{1}\left(s, \theta_{0}\right) d s \\
\int_{0}^{t} m_{2}\left(s, \theta_{0}\right) d s
\end{array}\right) \\
& +\left(\begin{array}{c}
-\alpha_{1} \mu_{1} \\
\alpha_{2} \mu_{2}
\end{array}\right)^{\top} V\left(\begin{array}{cc}
\frac{1}{\gamma_{1}}\left(e^{\gamma_{1} t}-1\right) & 0 \\
0 & \frac{1}{\gamma_{2}}\left(e^{\gamma_{2} t}-1\right)
\end{array}\right) V^{-1}\left(\begin{array}{c}
\lambda_{0}^{1} \\
\lambda_{0}^{2}
\end{array}\right) .
\end{aligned}
$$

The integrals $\int_{0}^{t} m_{1}\left(s, \theta_{0}\right) d s$ and $\int_{0}^{t} m_{2}\left(s, \theta_{0}\right) d s$ are respectively given by

$$
\begin{aligned}
\int_{0}^{t} m_{1}\left(s, \theta_{0}\right) d s & =e_{1}^{\top} M_{2}\left(t, \theta_{0}\right)\left(\begin{array}{c}
\frac{1}{\Upsilon} \kappa_{1}\left(\left(\delta_{1,1} \mu_{1}-\kappa_{1}\right)-\gamma_{2}\right) \\
\frac{1}{\Upsilon} \kappa_{2} \delta_{1,2} \mu_{2}
\end{array}\right) \\
\int_{0}^{t} m_{2}\left(s, \theta_{0}\right) d s & =e_{2}^{\top} M_{2}\left(t, \theta_{0}\right)\left(\begin{array}{c}
\frac{1}{\Upsilon} \kappa_{1}\left(\gamma_{1}-\left(\delta_{1,1} \mu_{1}-\kappa_{1}\right)\right) \\
-\frac{1}{\Upsilon} \kappa_{2} \delta_{1,2} \mu_{2}
\end{array}\right),
\end{aligned}
$$

where $M_{2}\left(t, \theta_{0}\right)$ is a time-dependent matrix defined by

$$
\begin{aligned}
& M_{2}\left(t, \theta_{0}\right)=\left(\begin{array}{cc}
\theta_{0}^{\top}\left(Q_{0}-\gamma_{1} I\right)^{-1} & 0 \\
0 & \theta_{0}^{\top}\left(Q_{0}-\gamma_{2} I\right)^{-1}
\end{array}\right) \\
& \times\left(\begin{array}{cc}
\left(Q_{0}\right)^{-1}\left(\exp \left(Q_{0} t\right)-I\right)-\frac{1}{\gamma_{1}} I\left(e^{\gamma_{1} t}-1\right) & 0 \\
0 & \left(Q_{0}\right)^{-1}\left(\exp \left(Q_{0} t\right)-I\right)-\frac{1}{\gamma_{2}} I\left(e^{\gamma_{2} t}-1\right)
\end{array}\right)\left(\begin{array}{ll}
c_{1} & c_{2} \\
c_{1} & c_{2}
\end{array}\right) .
\end{aligned}
$$

Notice that the matrice $Q_{0}$ is not well conditioned as the sum of its column is the null vector. In theory, it is then not possible to invert it. However, the expression 28 may be calculated if we remember the definition of the matrix exponential. In this case, we calculate $Q_{0}^{-1}\left(\exp \left(Q_{0} t\right)-I\right)$ by the following sum:

$$
\begin{aligned}
Q_{0}^{-1}\left(\exp \left(Q_{0} t\right)-I\right) & =Q_{0}^{-1}\left(I+\sum_{k=1}^{\infty} \frac{1}{k !} Q_{0}^{k} t^{k}-I\right) \\
& =\sum_{k=1}^{\infty} \frac{1}{k !} Q_{0}^{k-1} t^{k}
\end{aligned}
$$

From the last proposition, we infer that the long term mean of the stock price is constant if $\gamma_{1}<0$ and $\gamma_{2}<0$. In this case, the asymptotic stock price is constant and detailed in the next corollary: 
Corollary 3.8. If $\gamma_{1}$ and $\gamma_{2}$ are strictly negative, the asymptotic expected price is equal to:

$$
\begin{aligned}
\lim _{t \rightarrow \infty} \mathbb{E}\left(S_{t} \mid \mathcal{F}_{0}\right) & =S_{0}+\left(\begin{array}{c}
-\alpha_{1} \mu_{1} \\
\alpha_{2} \mu_{2}
\end{array}\right)^{\top} V \lim _{t \rightarrow \infty}\left(\begin{array}{c}
\int_{0}^{t} m_{1}\left(s, \theta_{0}\right) d s \\
\int_{0}^{t} m_{2}\left(s, \theta_{0}\right) d s
\end{array}\right) \\
& +\left(\begin{array}{c}
-\alpha_{1} \mu_{1} \\
\alpha_{2} \mu_{2}
\end{array}\right)^{\top} V\left(\begin{array}{cc}
-\frac{1}{\gamma_{1}} & 0 \\
0 & -\frac{1}{\gamma_{2}}
\end{array}\right) V^{-1}\left(\begin{array}{c}
\lambda_{0}^{1} \\
\lambda_{0}^{2}
\end{array}\right),
\end{aligned}
$$

where the limits of integrals present in the first term are given by

$$
\begin{aligned}
& \lim _{t \rightarrow \infty} \int_{0}^{t} m_{1}\left(s, \theta_{0}\right) d s=e_{1}^{\top} M_{2}\left(\infty, \theta_{0}\right)\left(\begin{array}{c}
\frac{1}{\Upsilon} \kappa_{1}\left(\left(\delta_{1,1} \mu_{1}-\kappa_{1}\right)-\gamma_{2}\right) \\
\frac{1}{\Upsilon} \kappa_{2} \delta_{1,2} \mu_{2}
\end{array}\right) \\
& \lim _{t \rightarrow \infty} \int_{0}^{t} m_{2}\left(s, \theta_{0}\right) d s=e_{2}^{\top} M_{2}\left(\infty, \theta_{0}\right)\left(\begin{array}{c}
\frac{1}{\Upsilon} \kappa_{1}\left(\gamma_{1}-\left(\delta_{1,1} \mu_{1}-\kappa_{1}\right)\right) \\
-\frac{1}{\Upsilon} \kappa_{2} \delta_{1,2} \mu_{2}
\end{array}\right),
\end{aligned}
$$

and where $M_{2}\left(\infty, \theta_{0}\right)$ is the constant matrix

$$
M_{2}\left(\infty, \theta_{0}\right)=\left(\begin{array}{ccc}
\theta_{0}^{\top}\left(Q_{0}-\gamma_{1} I\right)^{-1}\left(\left(Q_{0}\right)^{-1}(\Pi-I)+\frac{1}{\gamma_{1}} I\right) c_{1} & 0 \\
0 & \theta_{0}^{\top}\left(Q_{0}-\gamma_{2} I\right)^{-1}\left(\left(Q_{0}\right)^{-1}(\Pi-I)+\frac{1}{\gamma_{2}} I\right) c_{2}
\end{array}\right) .
$$

As mentioned in the introduction, we denote by $\psi_{1}($.$) and \psi_{2}($.$) the moment generating of O_{1}$ and $\mathrm{O}_{2}$. The next proposition presents the Laplace transform of the number of jumps $N^{k}, k \in\{1,2\}$ which is the exponential of an affine function of the intensities. This result is very useful if we want to calculate numerically the first moments of orders counting processes.

Proposition 3.9. For any $\omega \in \mathbb{R}$, the probability generating function for $N_{s}^{k}$ for $k=1,2$ with $s \geq t$ is given by

$$
\mathbb{E}\left(\omega^{N_{s}^{k}} \mid \mathcal{F}_{t}\right)=\omega^{N_{t}^{k}} \exp \left(A\left(t, s, \theta_{t}\right)+B_{k}(t, s) \lambda_{t}^{k}\right) \quad, k \in\{1,2\}
$$

where $B(t, s)$ is the solution of an ODE:

$$
\begin{aligned}
\frac{\partial}{\partial t} B_{1} & =\kappa_{1} B_{1}-\left[1_{k=1} \omega \psi_{1}\left(B_{1} \delta_{1,1}+B_{2} \delta_{2,1}\right)-1\right] \\
\frac{\partial}{\partial t} B_{2} & =\kappa_{2} B_{2}-\left[1_{k=2} \omega \psi_{2}\left(B_{1} \delta_{1,2}+B_{2} \delta_{2,2}\right)-1\right]
\end{aligned}
$$

with the terminal condition $B_{k}(s, s)=0$ for $k=1,2$. Let us define $\tilde{A}(t, s)=\left[e^{A\left(t, s, e_{1}\right)}, \ldots, e^{A\left(t, s, e_{N}\right)}\right]^{\top}$. $\tilde{A}(t, s)$ is a vector, solution of the ODE system:

$$
\frac{\partial \tilde{A}(t, s)}{\partial t}+\left(\operatorname{diag}\left(\kappa_{1} c_{1, t} B_{1}+\kappa_{2} c_{2, t} B_{2}\right)+Q_{0}\right) \tilde{A}(t, s)=0
$$

under the terminal boundary condition:

$$
\tilde{A}(T, T)=0_{N} .
$$

where $0_{N}$ is the null vector of dimension $N$. 
The proof is in appendix. The next proposition presents the moment generating function (mgf) of $S_{t}$. The mgf may be inverted numerically by a discrete Fourier transform to retrieve the probability density function of $S_{t}$. We could eventually think to use this density to calibrate the model by loglikelihood maximization. In numerical applications, we instead opt for a MCMC algorithm which is detailed in section 4.1 .

Proposition 3.10. For any $\left(\omega_{1}, \omega_{2}, \omega_{3}\right) \in \mathbb{C}_{-}{ }^{3}$, the mgf of $\omega_{1} S_{s}+\omega_{2} \lambda_{s}^{1}+\omega_{3} \lambda_{s}^{2}$ for $s \geq t$, is given by the following expression

$$
\mathbb{E}\left(e^{\omega_{1} S_{s}+\omega_{2} \lambda_{s}^{1}+\omega_{3} \lambda_{s}^{2}} \mid \mathcal{F}_{t}\right)=\exp \left(\omega_{1} S_{t}+A\left(t, s, \theta_{t}\right)+B_{1}(t, s) \lambda_{t}^{1}+B_{2}(t, s) \lambda_{t}^{2}\right),
$$

where $B_{1}(t, s)$ and $B_{2}(t, s)$ are functions of time, solutions of the ODE:

$$
\begin{aligned}
\frac{\partial}{\partial t} B_{1} & =\kappa_{1} B_{1}-\omega_{1} \alpha_{1} \mu_{1}-\left[\psi_{1}\left(B_{1} \delta_{1,1}+B_{2} \delta_{2,1}+C_{1}\right)-1\right] \\
\frac{\partial}{\partial t} B_{2} & =\kappa_{2} B_{2}+\omega_{1} \alpha_{2} \mu_{2}-\left[\psi_{2}\left(B_{1} \delta_{1,2}+B_{2} \delta_{2,2}+C_{2}\right)-1\right]
\end{aligned}
$$

with the terminal condition $B_{1}(s, s)=\omega_{2}$ and $B_{2}(s, s)=\omega_{3}$. And where

$$
\tilde{A}(t, s)=\left[e^{A\left(t, s, e_{1}\right)}, \ldots, e^{A\left(t, s, e_{N}\right)}\right]^{\top}
$$

is a vector of functions, solution of the ODE system:

$$
\frac{\partial \tilde{A}(t)}{\partial t}+\left(\operatorname{diag}\left(\kappa_{1} c_{1, t} B_{1}+\kappa_{2} c_{2, t} B_{2}\right)+Q_{0}\right) \tilde{A}(t)=0 .
$$

under the terminal boundary condition:

$$
\tilde{A}(T, T, j)=0 \quad j=1 \ldots N .
$$

$B_{1}(t, s)$ and $B_{2}(t, s)$ do not admit any simple analytical expression. However, they can be reformulated as solution of a non-linear system of equations. Furthermore, we can find the domain of $\mathbb{R}$, on which these functions are defined as stated in the next proposition.

Proposition 3.11. for $k=1,2$, let us define

$$
\beta_{k}\left(\omega_{1}\right)=(-1)^{k} \omega_{1} \alpha_{k} \mu_{k}+1 .
$$

and functions $F_{\omega_{1}}^{1}(x, y):<, \mathbb{R}^{2} \rightarrow \mathbb{R}^{+}$,

$$
\begin{aligned}
& F_{\omega_{1}}^{1}(x, y):=\int_{\omega_{2}}^{x} \frac{d u_{1}}{-\kappa_{1} u_{1}+\psi_{1}\left(u_{1} \delta_{1,1}+y \delta_{2,1}+C_{1}\right)-\beta_{1}\left(\omega_{1}\right)}, \\
& F_{\omega_{1}}^{2}(x, y):=\int_{\omega_{3}}^{y} \frac{d u_{2}}{-\kappa_{2} u_{2}+\psi_{2}\left(x \delta_{1,2}+u_{2} \delta_{2,2}+C_{2}\right)-\beta_{2}\left(\omega_{1}\right)} .
\end{aligned}
$$

If $\left(F_{\omega_{1}}^{1}\right)^{-1}(\tau \mid y)$ and $\left(F_{\omega_{1}}^{2}\right)^{-1}(\tau \mid x)$ are respectively the inverse functions of $F_{\omega_{1}}^{1}(., y)$ and $F_{\omega_{1}}^{2}(x,$.$) ,$ then

$$
\left\{\begin{array}{l}
B_{1}(t, s)=\left(F_{\omega_{1}}^{1}\right)^{-1}\left(s-t \mid B_{2}(t, s)\right) \\
B_{2}(t, s)=\left(F_{\omega_{1}}^{2}\right)^{-1}\left(s-t \mid B_{1}(t, s)\right)
\end{array}\right.
$$

And for $k \in\{1,2\}, B_{k} \in\left[\omega k+1, u_{k}^{*}\right)$ or $B_{k} \in\left[u_{k}^{*}, \omega k+1\right)$ where $\left(u_{1}^{*}, u_{2}^{*}\right)$ is the unique solution of the system:

$$
\psi_{k}\left(u_{1} \delta_{1, k}+u_{2} \delta_{2, k}+C_{k}\right)=\beta_{k}\left(\omega_{1}\right)+\kappa_{k} u_{k}
$$


In numerical applications, we prefer to solve numerically ODE's (34) instead of inverting functions $F_{\omega_{1}}^{1}$ and $F_{\omega_{1}}^{2}$, which reveals hard to numerically invert in practice.

\section{Estimation of parameters}

Given that economic regimes and jump intensities are not directly observable, the estimation of SMM parameters is challenging. On the other hand, the statistical distribution of prices does not admit a closed form expression. It is then not possible to infer parameters by log-likelihood maximization. Instead, we use a Particle Monte Carlo Markov Chain (PMCMC) method to fit the SMM to a time serie. The PMCMC algorithm is based on a particle filter that evaluates the log-likelihood by simulations. The next paragraph details this filter.

\subsection{A Particle filter}

The Markov chain $\theta_{t}$ and intensities of jumps $\lambda_{t}^{1}, \lambda_{t}^{2}$, are hidden state variables. We use then a sequential Monte-Carlo (SMC) method, also called particle filter, to guess their sample paths. This Bayesian technique is combined later with a Monte-Carlo Markov Chain to fit the SMM, but for the moment, we assume that parameters are known. The procedure is based on a discrete versions of equations (4) defining prices and (11) that drives the jumps arrival intensities. We denote by $\Delta$ the length of the time interval. The ex ante variation of prices (over the period $\Delta$ ) at time $t_{j}=j \Delta$, defined by $X_{j}=S_{(j+1) \Delta}-S_{j \Delta}$, then satisfies the following equation in discrete time

$$
X_{j}=\alpha_{2} \Delta L_{j}^{2}-\alpha_{1} \Delta L_{j}^{1},
$$

where $\Delta L_{j}^{i}=\sum_{u=N_{j \Delta}^{i}}^{N_{(j+1) \Delta}^{i}} O_{u}^{i}$ for $i=1,2$ is the sum of buy-sell orders. In the discretized framework, $N_{(j+1) \Delta}^{i}-N_{j \Delta}^{i}$ are Poisson random variables with a constant intensity $\lambda_{j}^{i} \Delta$, over $\Delta$. The economic regime is assumed to remain unchanged over the time interval $\Delta$ and the value of $\theta_{t}$ for $t \in[j \Delta,(j+1) \Delta]$ is denoted by $\theta_{j}$. The mean reversion levels of $\lambda_{j}^{1}$ and $\lambda_{j}^{2}$ are constant over the $j^{\text {th }}$ interval of time and equal to $c_{i, j}=c_{i}^{\top} \theta_{j}$ for $i=1,2$.

The Euler approximation of equations 11 provides the discrete dynamics of latent processes $\lambda^{i}=\left(\lambda_{t}^{i}\right)_{t}:$

$$
\lambda_{j+1}^{i}=\lambda_{j}^{i}+\kappa_{i}\left(c_{i, j}-\lambda_{j}^{i}\right) \Delta+\delta_{i, 1} \Delta L_{j}^{1}+\delta_{i, 2} \Delta L_{j}^{2} \quad i=1,2 .
$$

The second latent process carries the information about the economic regime. We denote by $\left(\theta_{j}\right)_{j \in \mathbb{N}}$ the discrete Markov chain approximating $\theta_{t}$. This chain has a matrix of transition probabilities denoted $P_{\Delta}=\exp \left(Q_{0} \Delta\right)$ and the transition random measure $K($.$) such that \theta_{j+1}=\int_{\theta \in E} K\left(\theta_{j}, d \theta\right)$. Remember that at this stage, the model parameters are assumed to be known. A particle at time $t_{j}$ is a triplet denoted by $v_{j}=\left(\lambda_{j}^{1}, \lambda_{j}^{2}, \theta_{j}\right)$ that contains information about the economic regime and intensities. The model admits a useful state-space representation, where the equation (37) provides a measurement equation or system (the 'space') that defines the relationship between variations of prices and hidden state variables. The particle $v_{j}$ helps to find the transition system (the 'state') that describes the dynamics of state variables.

In the remainder of the paper, we denote by $\left\{x_{1}, x_{2}, \ldots, x_{n}\right\}$, the sample of observed variations of stock prices, realisations of $X_{j}$ for $j=1, \ldots, n$. Conditionally to information contained in $v_{j}$, the 
probability density function (pdf) of price variations at time $j p\left(x_{j} \mid v_{j}\right)$ is given by

$$
\begin{aligned}
p\left(x_{j} \mid v_{j}\right)= & \sum_{k_{1}=1}^{\infty} \sum_{k_{2}=1}^{\infty}\left[P\left(N_{(j+1) \Delta}^{1}-N_{j \Delta}^{1}=k_{1} \mid \lambda_{j}^{1}\right)\right. \\
& \times P\left(N_{(j+1) \Delta}^{2}-N_{j \Delta}^{2}=k_{2} \mid \lambda_{j}^{2}\right) \times f_{k_{1}, k_{2}}\left(x_{j}\right) \\
& +P\left(N_{(j+1) \Delta}^{1}-N_{j \Delta}^{1}=0 \mid \lambda_{j}^{1}\right) \\
& \left.\times P\left(N_{(j+1) \Delta}^{2}-N_{j \Delta}^{2}=0 \mid \lambda_{j}^{2}\right) \mathbf{1}_{\left\{x_{j}=0\right\}}\right]
\end{aligned}
$$

where $f_{k_{1}, k_{2}}($.$) is the convoluted law of the sum of random variables: \alpha_{2} \sum_{i=1}^{k_{2}} O_{i}^{2}-\alpha_{1} \sum_{i=1}^{k_{1}} O_{i}^{1}$. Given that the interval $\Delta$ between two successive observations is small, the probability of observing more than one or two jumps is negligible. The sum in equation (39) may then be limited to a few terms in order to reduce the computation time. In numerical applications orders are assumed distributed as normal random variables and $\alpha_{1}, \alpha_{2}$ are set to one. The sum $\alpha_{2} \sum_{i=1}^{k_{2}} O_{i}^{2}-\alpha_{1} \sum_{i=1}^{k_{1}} O_{i}^{1}$ is then Gaussian with a mean and a standard deviation respectively equal to $\left(k_{2} \mu_{2}-k_{1} \mu_{1}\right)$ and $\sqrt{k_{2} \sigma_{2}^{2}+k_{1} \sigma_{1}^{2}}$. Here $\sigma_{1}^{2}$ and $\sigma_{2}^{2}$ are the variance of $O^{1}$ and $O^{2}$.

On the other hand, it is also possible to simulate the transition density $p\left(v_{j+1} \mid v_{j}\right)$ with equations 38 and $\theta_{j+1}=\int_{\theta \in E} K\left(\theta_{j}, d \theta\right)$. The density of the intial particle $v_{0}$ is denoted by $p\left(v_{0}\right)$ and the posterior distribution of $v_{j}$ given observations till time $t_{j}$, is denoted by $p\left(v_{j} \mid x_{1: j}\right)$. Using the Bayes' rule, the posterior distribution is developped as follows

$$
p\left(v_{j} \mid x_{1: j}\right)=\frac{p\left(x_{1: j}, v_{j}\right)}{p\left(x_{1: j}\right)}
$$

and the denominator satisfies the equality:

$$
p\left(x_{1: j}\right)=p\left(x_{1: j-1}, x_{j}\right)=p\left(x_{j} \mid x_{1: j-1}\right) p\left(x_{1: j-1}\right) .
$$

Given that the numerator of equation $(40)$ is equal to

$$
p\left(x_{1: j}, v_{j}\right)=p\left(x_{j} \mid v_{j}\right) p\left(v_{j} \mid x_{1: j-1}\right) p\left(x_{1: j-1}\right),
$$

the expression for the posterior distribution is rewritten as:

$$
p\left(v_{j} \mid x_{1: j}\right)=\frac{p\left(x_{j} \mid v_{j}\right)}{\int p\left(x_{j} \mid v_{j}\right) p\left(v_{j} \mid x_{1: j-1}\right) d v_{j}} p\left(v_{j} \mid x_{1: j-1}\right),
$$

where

$$
p\left(v_{j} \mid x_{1: j-1}\right)=\int p\left(v_{j} \mid v_{j-1}\right) p\left(v_{j-1} \mid x_{1: j-1}\right) d v_{j-1} .
$$

The calculation of $p\left(\lambda_{j}^{1}, \lambda_{j}^{2}, \theta_{j} \mid x_{1: j}\right)$ is done in two steps. The first one is a prediction step in which we estimate $p\left(v_{j} \mid x_{1: j-1}\right)$ by the relation 42 . In the correction step, we approach the probabilities $p\left(v_{j} \mid x_{1: j}\right)$ using the equation (41). In practice, the integral in the prediction step is replaced by a Monte Carlo simulation, of $M$ particles, denoted by $v_{j}^{(k)}=\left(\lambda_{j}^{1(k)}, \lambda_{j}^{2(k)}, \theta_{j}^{(k)}\right)$ for $k=1, \ldots, M$. The structure of the particle filter algorithm is the following: 


\section{Particle filter algorithm}

1. Initial step: draw $M$ values of $v_{0}^{(k)}$ for $k=1, \ldots, M$, from an initial distribution $p\left(v_{0}\right)$

2. For $j=1: T$

Prediction step: draw a sample of $\Delta L_{j}^{1(k)}, \Delta L_{j}^{2(k)}$ and $\theta_{j}^{(k)}$ and update $\lambda_{j}^{1(k)}, \lambda_{j}^{2(k)}, c_{j}^{1(k)}$, $c_{j}^{2(k)}$ using the relations $38 c_{j}^{1(k)}=c_{1}^{\top} \theta_{j}^{(k)}$, and $c_{j}^{2(k)}=c_{2}^{\top} \theta_{j}^{(k)}$.

Correction step: the particle $v_{j}^{(k)}$ has a probability of $w_{j}^{(k)}=\frac{p\left(x_{j} \mid v_{j}\right)}{\sum_{k=1: M} p\left(x_{j} \mid v_{j}^{(k)}\right)}$ where $p\left(x_{j} \mid v_{j}^{(k)}\right)$ is distributed according to the mixture distribution of equation $[39]$.

Resampling step: resample with replacement $M$ particles according to the importance weights $w_{j}^{(k)}$. The new importance weights are set to $w_{j}^{(k)}=\frac{1}{M}$.

Finally, the filtered intensities for the period $j$ is computed as the sum of particles, weighted by their probabilities of occurrence:

$$
\mathbb{E}\left(\lambda_{j}^{1} \mid \mathcal{G}_{T}\right)=\sum_{i=1: M} \lambda_{j}^{1(i)} w_{j}^{(k)} \quad \mathbb{E}\left(\lambda_{j}^{2} \mid \mathcal{G}_{T}\right)=\sum_{i=1: M} \lambda_{j}^{2(i)} w_{j}^{(k)},
$$

whereas the log-likelihood is approached as follows:

$$
\begin{aligned}
\log L(\Theta) & =\sum_{j=1}^{T} \log p\left(x_{j} \mid x_{j-1}\right) \\
& =\sum_{j=1}^{T} \log \int p\left(x_{j} \mid v_{j}\right) p\left(v_{j} \mid v_{j-1}\right) d v_{j} \\
& =\sum_{j=1}^{T} \log \left(\frac{1}{M} \sum_{k=1}^{M} p\left(x_{j} \mid v_{j}^{(k)}\right)\right) .
\end{aligned}
$$

However, the estimator of the likelihood is not continuous as a function of parameters because it is based on simulations. Fitting parameters by log-likelihood maximization is then inefficient. This observation justifies working with a Monte-Carlo Markov Chain algorithm.

\subsubsection{Application on simulated data}

To conclude this section, we test the performance of the SMC filter with a simulated data-set. We first simulate a daily sample path of a SMM, with three economic regimes and over a period of ten years. The parameters used for this simulation 1 are reported in table 1. The first state corresponds to a period of economic recession: negative average return, high volatility and frequency of jumps. The third regime represents a period of economic growth: positive expected return, low volatility and frequency of jumps. The second state is an intermediate conjuncture, close to economic stagnation. The one year matrix of transition probabilities used for this exercise is presented in table 2

\footnotetext{
${ }^{1}$ Chosen parameters are in the same range of values as real estimates reported in section 4.2 In order to clearly vizualize changes of regimes, the gap between mean reversion levels in each regimes is increased. For the same reason, we have also modified transition probabilities in order to observe a sufficient number of changes of regime during the simulation.
} 


\begin{tabular}{cccccccc}
\hline \hline$c_{1,1}$ & 10 & $c_{2,1}$ & 20 & $\kappa_{1}$ & 56 & $\kappa_{2}$ & 50 \\
$c_{1,2}$ & 35 & $c_{2,2}$ & 70 & $\alpha_{1}$ & 1 & $\alpha_{2}$ & 1 \\
$c_{1,3}$ & 50 & $c_{2,3}$ & 100 & $\mu_{1}$ & 10 & $\mu_{2}$ & 10 \\
$\delta_{1,1}$ & 1 & $\delta_{2,1}$ & 1 & $\sigma_{1}$ & 5 & $\sigma_{2}$ & 3 \\
$\delta_{1,2}$ & 1 & $\delta_{2,2}$ & 1 & $\lambda_{0}^{1}$ & 12 & $\lambda_{0}^{2}$ & 17 \\
\hline \hline
\end{tabular}

Table 1: This table reports the parameters used for the simulation of a daily sample path of the SMM, with three regimes.

\begin{tabular}{cccc}
\hline \hline$\left(p_{i j}(0,1)\right)_{i, j=1,2,3}$ & state 1 & state 2 & state 3 \\
state 1 & 0.60 & 0.20 & 0.20 \\
state 2 & 0.20 & 0.60 & 0.20 \\
state 3 & 0.25 & 0.25 & 0.50 \\
\hline \hline
\end{tabular}

Table 2: This table presents the one year matrix of transition of $\theta_{t}$, used for the simulation of a daily sample path of the SMM, with three regimes.
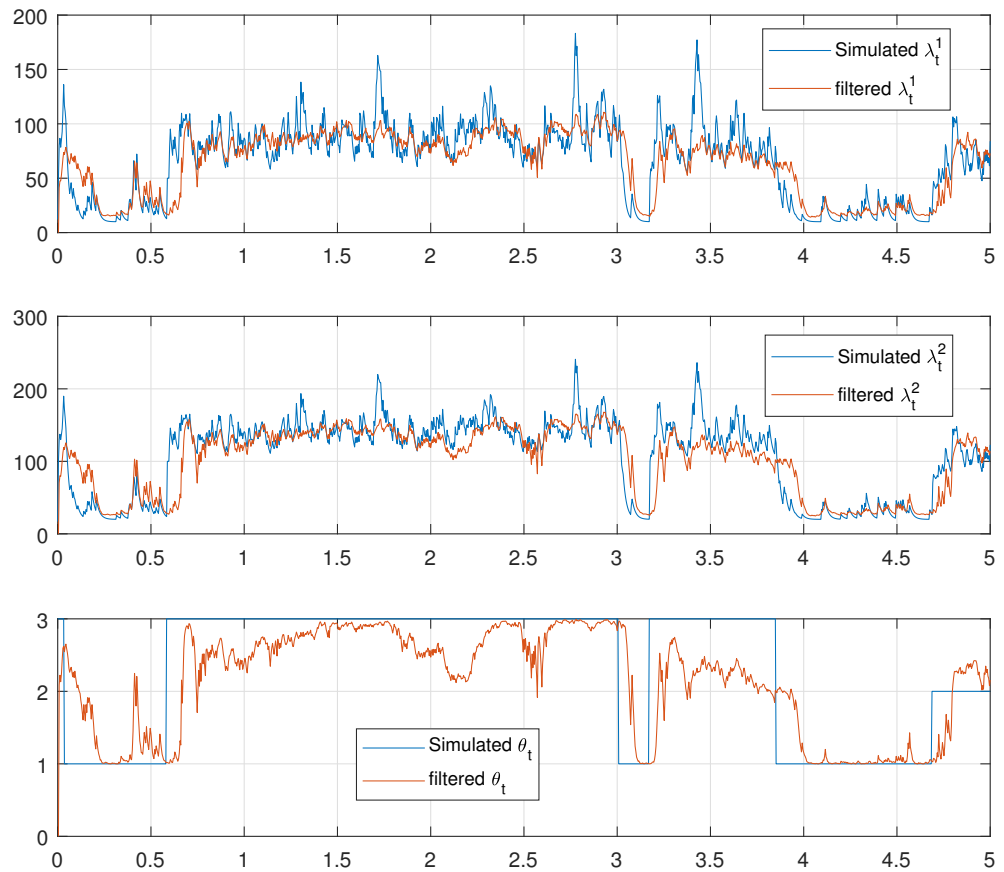

Figure 1: This graph shows simulated and filtered sample paths of $\lambda_{t}^{1}, \lambda_{t}^{2}$ and $\theta_{t}$.

After simulation of a sample path, we run the SMC filter with 500 particles. The graphs of 
figure 1 compare simulated and filtered intensities of jumps and economic regimes. They confirm the efficiency of the SMC algorithm. This filter is combined in a next section to a Monte Carlo Markov Chain (MCMC) algorithm to estimate the SSEJD. But before, we introduce an approached estimation method that is used to initialize the MCMC algorithm.

\subsection{Calibration by Particle Monte Carlo Markov Chain}

When dealing with a non-Gaussian and nonlinear specification, simulation-based methods offer strong advantages over the alternative approaches. In this paper, we employ a Particle Markov Chain Monte Carlo method (PMCMC) to fit the SMM to a time-serie. We refer to Doucet et al. (2000) for a review of other simulation-based methods. The set of parameters is denoted by $\Xi$ and serves us as index for the probability distribution function. We adopt a Bayesian approach to estimate $\Xi$ by computing the parameters posterior distribution

$$
\pi(\Xi)=p\left(\Xi \mid x_{1: T}\right)=\frac{p(\Xi) p\left(x_{1: T} \mid \Xi\right)}{\int p\left(\Xi^{\prime}\right) p\left(x_{1: T} \mid \Xi^{\prime}\right) d \Xi^{\prime}}
$$

where $p(\Xi)$ and $p\left(x_{1 \ldots T} \mid \Xi\right)$ denotes respectively the parameters prior distribution and the likelihood of the data. The density $\pi(\Xi)$ is built by the PMCMC method that generates a sample from $\pi(\Xi)$ by creating a Markov chain with the same stationary distribution as the parameters posterior one. Once that the Markov chain has reached stationarity after a transient phase, called "burn-in" period, samples from the posterior distribution can then be simulated. Standard MCMC algorithm requires a point-wise estimate of $p\left(x_{1 \ldots T} \mid \Xi\right)$, that is not available in our model. Instead, $p\left(x_{1 \ldots T} \mid \Xi\right)$ is approached by its estimate computed with a particle filter.

The construction of the Markov chain consists of two steps, iteratively repeated. At the beginning of the $k^{\text {th }}$ iteration, we propose a candidate parameter $\Xi^{\prime}$ from a proposal distribution $q\left(\Xi^{\prime} \mid \Xi^{(k-1)}\right)$ given the previous state of the Markov chain, noted $\Xi^{(k-1)}$. The proposal distribution has a support that covers the target distribution. In the second step, we determine if we update the state by $\Xi^{\prime}$. For this purpose, the acceptance probability is computed as follows

$$
\varepsilon\left(\Xi^{\prime}, \Xi^{(k-1)}\right)=\min \left\{1, \frac{\pi\left(\Xi^{\prime}\right)}{\pi\left(\Xi^{(k-1)}\right)} \frac{q\left(\Xi^{(k-1)} \mid \Xi^{\prime}\right)}{q\left(\Xi^{\prime} \mid \Xi^{(k-1)}\right)}\right\} .
$$

This determines the probability that we assign the candidate parameter as the next state of the Markov chain, $\Xi^{\prime} \rightarrow \Xi^{(k)}$. Intuitively, if we disregard the influence of the proposal $q$, a candidate is accepted if it increases the posterior likelihood $\pi\left(\Xi^{\prime}\right)>\pi\left(\Xi^{(k-1)}\right)$. The presence of $q($.$) in equation$ (45) allows a small decrease in the posterior likelihood, so as to explore the entire posterior.

The resulting $K$ samples $\Xi^{(1: K)}$ (after the burn in period) serve next to build the empirical distribution of $\pi(\Xi)$, which is defined by

$$
\hat{\pi}(\Xi)=\frac{1}{K} \sum_{k=1}^{K} \delta_{\Xi^{(k)}}(d \Xi)
$$

where $\delta_{\Xi^{(k)}}(d \Xi)$ are the Dirac atoms located at $\Xi=\Xi^{(k)}$, with equal weights. The expected parameters with respect to the posterior distribution of parameters is then approached as follows

$$
\mathbb{E}\left(\Xi \mid x_{1: T}\right) \approx \frac{1}{K} \sum_{k=1: K} \hat{\pi}\left(\Xi^{(k)}\right) \Xi^{(k)} .
$$


In numerical applications, the transition distribution $q\left(\Xi^{\prime} \mid \Xi^{(k-1)}\right)$ is assumed Normal, $\mathcal{N}\left(\Xi^{\prime} \mid \Xi^{(k-1)}, \sigma_{q}\right)$. As this distribution is symmetric, $q\left(\Xi^{(k-1)} \mid \Xi^{\prime}\right)=q\left(\Xi^{\prime} \mid \Xi^{(k-1)}\right)$, the acceptance probability simplifies to

$$
\begin{aligned}
\varepsilon\left(\Xi^{\prime}, \Xi^{(k-1)}\right) & =\min \left\{1, \frac{\pi\left(\Xi^{\prime}\right)}{\pi\left(\Xi^{(k-1)}\right)}\right\}, \\
& =\min \left\{1, \frac{p\left(\Xi^{\prime}\right) p\left(x_{1: T} \mid \Xi^{\prime}\right)}{p\left(\Xi^{(k-1)}\right) p\left(x_{1: T} \mid \Xi^{(k-1)}\right)}\right\} .
\end{aligned}
$$

We use the PMCMC algorithm to calibrate the SMM model. We test the calibration algorithm with daily data of the S\&P 500, from February 2010 to February 2017 (1763 observations). We use this dataset to compare the classic approach without modulation of parameters, to models with 2 and 3 regimes. The PMCMC algorithm is applied to the set of parameters

$$
\Xi=\left\{\bar{c}, \kappa_{1}, \kappa_{2}, \mu_{1}, \mu_{2}, \sigma_{1}, \sigma_{2},\left(\delta_{i, j}\right)_{i, j=1,2},\left(q_{i, j}\right)_{i, j=1,2}\right\}
$$

that counts respectively 12, 16 and 22 parameters with 1, 2 and 3 regimes. Gatumel and Ielpo (2014) reject the hypothesis that two regimes are enough to capture asset returns evolutions for many securities. Their empirical results point out that between two and three regimes are required to capture the features of asset's distribution.

The filter runs with 500 particles and we perform 5000 iterations for the PMCMC procedure. We obtain acceptance rates of $36.41 \%$ and $41.16 \%$ for models with respectively 2 and 3 regimes. The convergence is checked by analyzing the log-likelihood, which is stable for both models after a burn in period of 2500 iterations. The average log-likelihoods over the last 2500 runs are reported in table 3. We also report the Akaike and Bayesian information criterions. These figures clearly confirm that switching models outperform the classic microstructure model with a single regime.

\begin{tabular}{cccc}
\hline \hline & $1 \mathrm{D}$ & $2 \mathrm{D}$ & $3 \mathrm{D}$ \\
\hline Log-likelihood & -7242 & -7119 & -7049 \\
AIC & 7266 & 7151 & 7093 \\
BIC & 14574 & 14358 & 14264 \\
\hline \hline
\end{tabular}

Table 3: Log-likelihood, Akaike Information Criterion (AIC), Bayesian Information Criterion (BIC)

Parameters estimated by the PMCMC algorithm are reported in tables 4 , 5 and 6 . The speeds of mean reversion $\left(\kappa_{1}, \kappa_{2}\right)$ are comparable for all models. In the 1D and 3D SMM, the parameters of mutual-excitation $\left(\delta_{12}, \delta_{21}\right)$ are less important than these of self-excitation $\left(\delta_{11}, \delta_{22}\right)$. For the $2 \mathrm{D}$ model, the situation is different and mutual excitation is more pronounced than the self-excitation. Averages and standard deviations of orders are similar whatever the model. Figures reported in table 5 reveals that the probabilities of staying in the same state over a period of one year are respectively around $56 \%$ and $36 \%$ for the $2 \mathrm{D}$ and $3 \mathrm{D}$ SMMs.

Table 6 compares the reversion levels of intensities in each regime. In the one dimension model, these levels for the supply and demand are comparable. In the $2 \mathrm{D} \mathrm{SMM}, c_{1,1}, c_{1,2}$ are respectively higher and lower than $c_{2,1}, c_{2,2}$. This means that the market receives more bid than ask orders in the first regime, and more ask than bid orders in the second regime. As bid and ask orders respectively 
drive down and up the stock price, the first regime is then assimilated to the conditions of a bear market. Whereas the second regime corresponds to a period of economic growth.

\begin{tabular}{ccccccc}
\hline \hline & \multicolumn{2}{c}{$1 \mathrm{D}$} & \multicolumn{2}{c}{$2 \mathrm{D}$} & \multicolumn{2}{c}{$3 \mathrm{D}$} \\
\hline & Estimate & St.dev. & Estimate & St.dev. & Estimate & St.dev. \\
\hline$\kappa_{1}$ & 21.316 & 2.790 & 25.802 & 5.283 & 43.187 & 4.765 \\
$\kappa_{2}$ & 38.238 & 4.678 & 33.828 & 6.010 & 38.408 & 4.289 \\
$\delta_{11}$ & 2.881 & 0.347 & 0.891 & 0.367 & 8.033 & 0.915 \\
$\delta_{12}$ & 0.2580 & 0.223 & 5.136 & 1.035 & 0.190 & 0.250 \\
$\delta_{21}$ & 0.132 & 0.152 & 4.512 & 0.532 & 0.184 & 0.188 \\
$\delta_{22}$ & 7.951 & 0.615 & 0.360 & 0.316 & 6.962 & 0.641 \\
$\mu_{1}$ & 6.708 & 0.403 & 5.220 & 0.255 & 4.880 & 0.303 \\
$\mu_{2}$ & 4.065 & 0.362 & 4.530 & 0.424 & 4.986 & 0.340 \\
$\sigma_{1}$ & 2.133 & 0.712 & 3.726 & 0.489 & 4.742 & 1.155 \\
$\sigma_{2}$ & 5.723 & 0.430 & 3.962 & 0.443 & 3.271 & 0.409 \\
\hline \hline
\end{tabular}

Table 4: This table reports parameters independent from $\theta_{t}$, for the 1D, 2D and 3D MSM models, (averages and standard deviations over the last 2500 simulations).

\begin{tabular}{cc}
\hline \hline & Transition matrix of probabilities \\
\hline 2D & $P=\left(\begin{array}{ll}0.572 & 0.428 \\
0.433 & 0.567\end{array}\right)$ \\
3D & $P=\left(\begin{array}{lll}0.369 & 0.296 & 0.335 \\
0.333 & 0.333 & 0.334 \\
0.333 & 0.299 & 0.368\end{array}\right)$ \\
\hline \hline
\end{tabular}

Table 5: Matrix of transition probabilities, for the SMM models with 2 and 3 regimes.

\begin{tabular}{ccccccc}
\hline \hline & & Estimate & St.dev. & & Estimate & St.dev. \\
\hline 1D & $c_{1}$ & 27.334 & 6.5064 & $c_{2}$ & 23.203 & 3.0661 \\
& & & & & & \\
$2 \mathrm{D}$ & $c_{1,1}$ & 48.858 & 9.741 & $c_{2,1}$ & 20.896 & 4.3289 \\
& $c_{1,2}$ & 21.43 & 5.0021 & $c_{2,2}$ & 32.234 & 7.087 \\
& & & & & & \\
$3 \mathrm{D}$ & $c_{1,1}$ & 27.035 & 6.2039 & $c_{2,2}$ & 2.4002 & 1.5993 \\
& $c_{1,2}$ & 11.852 & 2.4269 & $c_{2,2}$ & 27.471 & 7.2078 \\
& $c_{1,3}$ & 15.459 & 3.5566 & $c_{2,3}$ & 7.6532 & 4.9848 \\
\hline \hline
\end{tabular}

Table 6: Mean reversion levels of intensities in each regime for the three tested models. 

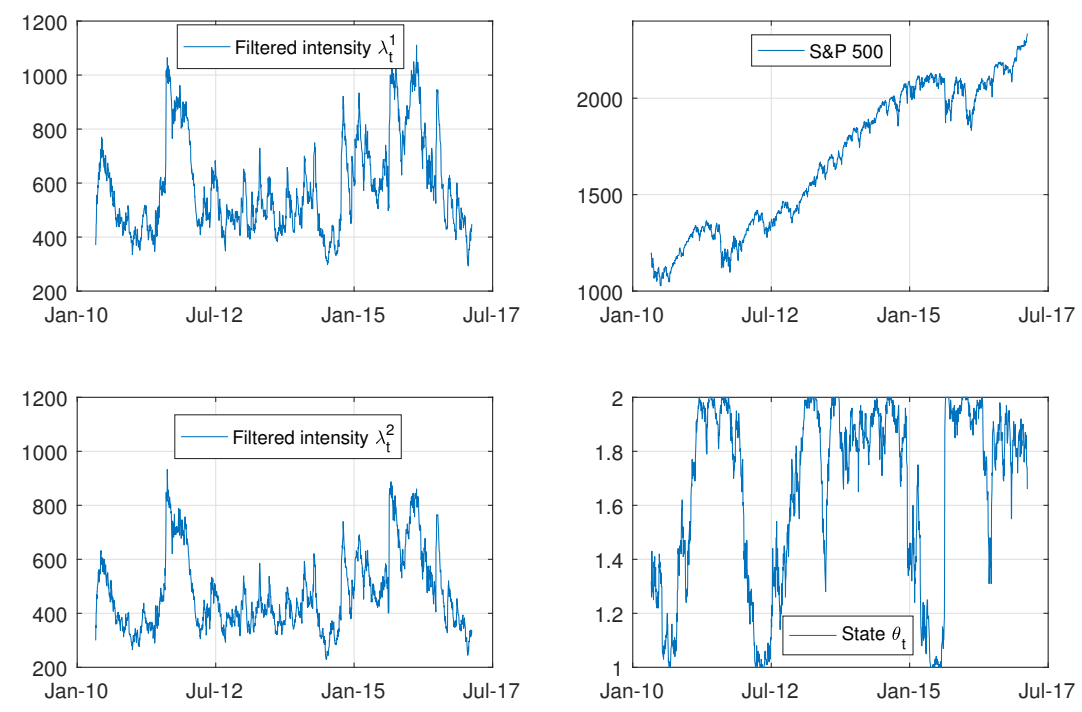

Figure 2: This graph shows filtered sample paths of $\lambda_{t}^{1}, \lambda_{t}^{2}$ and $\theta_{t}$ for the model with 2 regimes. The upper right plot presents the history of the S\&P 500 from 2010 to 2017.

This interpretation is confirmed by the right graphs of figure 2 that display the evolution of the $\mathrm{S} \& \mathrm{P}$ index 500 and filtered states. We observe a switch toward the first regime when the index drops. We draw the same conclusion for the 3D SMM, in which states one and two correspond respectively to bear and bull markets. Whereas the third regime is an intermediate state in which stock prices stagnate. The filtered regime informs us about the mood of markets. We may then imagine to use this information to define a dynamic trading strategy as proposed in Hainaut and MacGilchrist (2012). Finally, the two left graphs of figure 2 exhibit the filtered sample path of $\lambda_{t}^{1}$ and $\lambda_{t}^{2}$. We observe that intensities reach their highest level when the S\&P 500 falls. Monitoring these intensities could then be used by regulators to measure the stocks market stress.

\section{Conclusions}

This article proposes a new microstructure model for stock prices with regime shifts and mutualexcitation in the dynamic of orders arrivals. In this approach, called the switching microstructure model (SMM), the intensities of orders counting processes revert to a mean level that is modulated by a hidden Markov chain. This chain determines the direction of the market trend and the trading behaviour. In the first part of this work, we study the mathematical properties of the SMM. We show that the SMM presents a sufficient degree of analytical tractability for most of applications. The rest of the article focuses on the estimation of parameters.

The probability density function of prices does not have a closed form expression and increments of prices are not identically, independently distributed. Furthermore, prices depend upon three hidden state variables: the two mutually excited intensities of orders counting processes and the Markov chain. It is then not possible to estimate the SMM parameters by log-likelihood maximization. Instead, we develop a new sequential Monte Carlo algorithm to filter hidden processes, that is combined with a Markov Chain Monte Carlo (MCMC) procedure to estimate parameters. 
The model is next fitted to daily returns of the S\&P 500 stock index. This exercise reveals that the SMM with two and three regimes have a better explanatory power than a model without regime shift. Each state of the hidden Markov chain clearly corresponds to a particular trading trend. In the 3 states model, two regimes respectively correspond to a bear and a bull market whereas stock prices stagnate in the third regime. Filtering the evolution of the Markov chain can then help traders to adjust their positions to take advantage of market conditions. The filtered intensities of orders counting processes are also excellent indicators of markets stress.

\section{Appendix}

Proof of lemma 2.1 To prove this relation, it suffices to differentiate the expression of $\lambda_{t}^{i}$ to retrieve its dynamic:

$$
\begin{aligned}
d \lambda_{t}^{i}= & \kappa_{i} c_{i, t}-\kappa_{i}\left(\lambda_{0}^{i}-\kappa_{i} \int_{0}^{t} e^{\kappa_{i}(s-t)}\left(\lambda_{0}^{i}-c_{i, s}\right) d s+\right. \\
& \left.\int_{0}^{t} \delta_{i, 1} e^{\kappa_{i}(s-t)} d L_{s}^{1} d t+\int_{0}^{t} \delta_{i, 2} e^{\kappa_{i}(s-t)} d L_{s}^{2}\right)+\delta_{i, 1} d L_{t}^{1}+\delta_{i, 2} d L_{t}^{2} \\
= & \kappa_{i}\left(c_{i, t}-\lambda_{t}^{i}\right) d t+\delta_{i, 1} d L_{t}^{1}+\delta_{i, 2} d L_{t}^{2} \quad i=1,2 .
\end{aligned}
$$

To prove the positivity, we first remind that $\int_{0}^{t} \delta_{i, 1} e^{\kappa_{i}(s-t)} d L_{s}^{1}$ and $\int_{0}^{t} \delta_{i, 2} e^{\kappa_{i}(s-t)} d L_{s}^{2}$ are positive by construction. According to equation 12 , the process $\lambda_{t}^{i}$ admit the following lower bound:

$$
\lambda_{t}^{i}>\lambda_{0}^{i}+\left(\min \left(c_{i}\right)-\lambda_{0}^{i}\right) \kappa_{i} \int_{0}^{t} e^{\kappa_{i}(s-t)} d s .
$$

Given that $\kappa_{i} \int_{0}^{t} e^{\kappa_{i}(s-t)} d s=\left(1-e^{-\kappa_{i} t}\right)>0$, we conclude that

$$
\lambda_{t}^{i}>\lambda_{0}^{i} e^{-\kappa_{i} t}+\min \left(c_{i}\right)\left(1-e^{-\kappa_{i} t}\right)>0 .
$$

Proof of proposition 3.1. As $\mathcal{F}_{s} \subset \mathcal{F}_{s} \vee \mathcal{G}_{t}$, using nested expectations leads to the following expression for the expected intensity:

$$
\mathbb{E}\left(\lambda_{t}^{i} \mid \mathcal{F}_{s}\right)=\mathbb{E}\left(\mathbb{E}\left(\lambda_{t}^{i} \mid \mathcal{F}_{s} \vee \mathcal{G}_{t}\right) \mid \mathcal{F}_{s}\right)
$$

If we remember the expression (13) of the intensity, using the Fubini's theorem leads to the following expression for the expectation of $\lambda_{t}^{i}$, conditionally to the augmented filtration $\mathcal{F}_{s} \vee \mathcal{G}_{t}$ :

$$
\begin{aligned}
\mathbb{E}\left(\lambda_{t}^{i} \mid \mathcal{F}_{s} \vee \mathcal{G}_{t}\right)= & \lambda_{s}^{i}-\kappa_{i} \int_{s}^{t} e^{\kappa_{i}(u-t)}\left(\lambda_{s}^{i}-c_{i, u}\right) d u \\
& +\int_{s}^{t} \delta_{i, 1} e^{\kappa_{i}(u-t)} \mathbb{E}\left(d L_{u}^{1} \mid \mathcal{F}_{s} \vee \mathcal{G}_{t}\right)+\int_{s}^{t} \delta_{i, 2} e^{\kappa_{i}(u-t)} \mathbb{E}\left(d L_{u}^{2} \mid \mathcal{F}_{s} \vee \mathcal{G}_{t}\right)
\end{aligned}
$$

As the size of a jump is independent from the realized intensity before this jump, the expectation of $d L_{u}^{i}$ is the product of the average order size times the expected intensity:

$$
\mathbb{E}\left(d L_{u}^{i} \mid \mathcal{F}_{s} \vee \mathcal{G}_{t}\right)=\mu_{i} \times \mathbb{E}\left(\lambda_{u-}^{i} \mid \mathcal{F}_{s} \vee \mathcal{G}_{t}\right) d u \quad \forall u \leq t
$$


If we derive this last expression with respect to time, we find that $\mathbb{E}\left(\lambda_{t}^{i} \mid \mathcal{F}_{s} \vee \mathcal{G}_{t}\right)$ is solution of an ordinary differential equation (ODE):

$$
\begin{aligned}
\frac{\partial}{\partial t} \mathbb{E} & \left(\lambda_{t}^{i} \mid \mathcal{F}_{s} \vee \mathcal{G}_{t}\right)=-\kappa_{i}\left(\lambda_{s}^{i}-c_{i, t}\right)+\kappa_{i}^{2} \int_{s}^{t} e^{\kappa_{i}(u-t)}\left(\lambda_{s}-c_{i, u}\right) d u \\
& +\delta_{i, 1} \mu_{1} \mathbb{E}\left(\lambda_{t}^{1} \mid \mathcal{F}_{s} \vee \mathcal{G}_{t}\right)-\kappa_{i} \delta_{i, 1} \mu_{1} \int_{s}^{t} e^{-\kappa_{i}(t-u)} \mathbb{E}\left(\lambda_{u-}^{1} \mid \mathcal{F}_{s} \vee \mathcal{G}_{t}\right) d u \\
& +\delta_{i, 2} \mu_{2} \mathbb{E}\left(\lambda_{t}^{2} \mid \mathcal{F}_{s} \vee \mathcal{G}_{t}\right)-\kappa_{i} \delta_{i, 2} \mu_{2} \int_{s}^{t} e^{-\kappa_{i}(t-u)} \mathbb{E}\left(\lambda_{u-}^{2} \mid \mathcal{F}_{s} \vee \mathcal{G}_{t}\right) d u
\end{aligned}
$$

Using equation ( $(47))$, allows us to rewrite these ODE's as follows:

$$
\left(\begin{array}{l}
\frac{\partial}{\partial t} \mathbb{E}\left(\lambda_{t}^{1} \mid \mathcal{F}_{s} \vee \mathcal{G}_{t}\right) \\
\frac{\partial}{\partial t} \mathbb{E}\left(\lambda_{t}^{2} \mid \mathcal{F}_{s} \vee \mathcal{G}_{t}\right)
\end{array}\right)=\left(\begin{array}{c}
\kappa_{1} c_{1, t} \\
\kappa_{2} c_{2, t}
\end{array}\right)+\left(\begin{array}{cc}
\delta_{1,1} \mu_{1}-\kappa_{1} & \delta_{1,2} \mu_{2} \\
\delta_{2,1} \mu_{1} & \delta_{2,2} \mu_{2}-\kappa_{2}
\end{array}\right)\left(\begin{array}{l}
\mathbb{E}\left(\lambda_{t}^{1} \mid \mathcal{F}_{s} \vee \mathcal{G}_{t}\right) \\
\mathbb{E}\left(\lambda_{t}^{2} \mid \mathcal{F}_{s} \vee \mathcal{G}_{t}\right)
\end{array}\right)
$$

Solving this system of equation requires to determine eigenvalues $\gamma$ and eigenvectors $\left(v_{1}, v_{2}\right)$ of the matrix present in the right term of this system:

$$
\left(\begin{array}{cc}
\left(\delta_{1,1} \mu_{1}-\kappa_{1}\right) & \delta_{1,2} \mu_{2} \\
\delta_{2,1} \mu_{1} & \left(\delta_{2,2} \mu_{2}-\kappa_{2}\right)
\end{array}\right)\left(\begin{array}{l}
v_{1} \\
v_{2}
\end{array}\right)=\gamma\left(\begin{array}{l}
v_{1} \\
v_{2}
\end{array}\right)
$$

We know that eigenvalues cancel the determinant of the following matrix:

$$
\operatorname{det}\left(\begin{array}{cc}
\left(\delta_{1,1} \mu_{1}-\kappa_{1}\right)-\gamma & \delta_{1,2} \mu_{2} \\
\delta_{2,1} \mu_{1} & \left(\delta_{2,2} \mu_{2}-\kappa_{2}\right)-\gamma
\end{array}\right)=0,
$$

and are solutions of the second order equation:

$$
\gamma^{2}-\gamma\left(\left(\delta_{1,1} \mu_{1}-\kappa_{1}\right)+\left(\delta_{2,2} \mu_{2}-\kappa_{2}\right)\right)+\left(\delta_{1,1} \mu_{1}-\kappa_{1}\right)\left(\delta_{2,2} \mu_{2}-\kappa_{2}\right)-\delta_{1,2} \delta_{2,1} \mu_{1} \mu_{2}=0
$$

Roots of this last equation are $\gamma_{1}$ and $\gamma_{2}$, as defined by the equation 14. One way to find an eigenvector is to note that it must be orthogonal to each rows of the matrix:

$$
\left(\begin{array}{cc}
\left(\delta_{1,1} \mu_{1}-\kappa_{1}\right)-\gamma & \delta_{1,2} \mu_{2} \\
\delta_{2,1} \mu_{1} & \left(\delta_{2,2} \mu_{2}-\kappa_{2}\right)-\gamma
\end{array}\right)\left(\begin{array}{l}
v_{1} \\
v_{2}
\end{array}\right)=0
$$

then necessary,

$$
\left(\begin{array}{c}
v_{1}^{i} \\
v_{2}^{i}
\end{array}\right)=\left(\begin{array}{c}
-\delta_{1,2} \mu_{2} \\
\left(\delta_{1,1} \mu_{1}-\kappa_{1}\right)-\gamma_{i}
\end{array}\right) \quad \text { for } i=1,2 .
$$

If we note $D:=\operatorname{diag}\left(\gamma_{1}, \gamma_{2}\right)$, the matrix in the right term of equation (48) admits the decomposition:

$$
\left(\begin{array}{cc}
\delta_{1,1} \mu_{1}-\kappa_{1} & \delta_{1,2} \mu_{2} \\
\delta_{2,1} \mu_{1} & \delta_{2,2} \mu_{2}-\kappa_{2}
\end{array}\right)=V D V^{-1}
$$

where $V$ is the matrix of eigenvectors, as defined in equation (16). Its determinant, $\Upsilon$, and its inverse are respectively provided by equations $(18)$ and (17). If two new variables are defined as follows:

$$
\left(\begin{array}{l}
u_{1} \\
u_{2}
\end{array}\right)=V^{-1}\left(\begin{array}{l}
m_{1} \\
m_{2}
\end{array}\right) \text {. }
$$

The system 48 is decoupled into two independent ODEs:

$$
\frac{\partial}{\partial t}\left(\begin{array}{l}
u_{1} \\
u_{2}
\end{array}\right)=V^{-1}\left(\begin{array}{c}
\kappa_{1} c_{1, t} \\
\kappa_{2} c_{2, t}
\end{array}\right)+\left(\begin{array}{cc}
\gamma_{1} & 0 \\
0 & \gamma_{2}
\end{array}\right)\left(\begin{array}{l}
u_{1} \\
u_{2}
\end{array}\right) .
$$


And introducing the following notations

$$
V^{-1}\left(\begin{array}{c}
\kappa_{1} c_{1, t} \\
\kappa_{2} c_{2, t}
\end{array}\right)=\left(\begin{array}{c}
\epsilon_{1}(t) \\
\epsilon_{2}(t)
\end{array}\right)
$$

leads to the solutions for the system 49 :

$$
\left(\begin{array}{l}
u_{1}(t) \\
u_{2}(t)
\end{array}\right)=\left(\begin{array}{l}
\int_{s}^{t} \epsilon_{1}(u) e^{\gamma_{1}(t-u)} d u \\
\int_{s}^{t} \epsilon_{2}(u) e^{\gamma_{2}(t-u)} d u
\end{array}\right)+\left(\begin{array}{cc}
e^{\gamma_{1}(t-s)} & 0 \\
0 & e^{\gamma_{2}(t-s)}
\end{array}\right) V^{-1}\left(\begin{array}{c}
\lambda_{s}^{1} \\
\lambda_{s}^{2}
\end{array}\right)
$$

that allows us to infer the expression 15 for moments of $\lambda_{t}^{i}$. Notice that the determinant $\Upsilon$ is always real and if parameters of mutual excitations $\delta_{1,2}, \delta_{2,1}$, are positive. As $\mu_{1}, \mu_{2}>0$, the determinant is also strictly positive and the matric $V$ is invertible. Finally, equation (15) states that conditonally to the sample path of the Markov chain $\theta_{t}$, processes $\lambda_{t}^{1}$ and $\lambda_{t}^{2}$ are Markov given that their $\mathcal{F}_{s} \vee \mathcal{G}_{t}$-expectations only depend on the pair $\left(\lambda_{t}^{1}, \lambda_{t}^{2}\right)$

Proof of proposition 3.2 From the previous proposition, we infer that the unconditional expectations of OAI are the solutions of the following system

$$
\begin{aligned}
\left(\begin{array}{c}
\mathbb{E}\left(\lambda_{t}^{1} \mid \mathcal{F}_{s}\right) \\
\mathbb{E}\left(\lambda_{t}^{2} \mid \mathcal{F}_{s}\right)
\end{array}\right)= & V \int_{s}^{t}\left(\begin{array}{cc}
e^{\gamma_{1}(t-u)} & 0 \\
0 & e^{\gamma_{2}(t-u)}
\end{array}\right) V^{-1}\left(\begin{array}{c}
\kappa_{1} \mathbb{E}\left(c_{1, u} \mid \mathcal{F}_{s}\right) \\
\kappa_{2} \mathbb{E}\left(c_{2, u} \mid \mathcal{F}_{s}\right)
\end{array}\right) d u \\
& +V\left(\begin{array}{cc}
e^{\gamma_{1}(t-s)} & 0 \\
0 & e^{\gamma_{2}(t-s)}
\end{array}\right) V^{-1}\left(\begin{array}{c}
\lambda_{s}^{1} \\
\lambda_{s}^{2}
\end{array}\right) .
\end{aligned}
$$

Given that $\theta_{t}$ is a finite state Markov chain of generator $Q_{0}$ and if we remember that $c_{i}=\left(\begin{array}{c}c_{i, 1} \\ \vdots \\ c_{i, N}\end{array}\right)$ for $i=1,2$ are $N$ vectors, the expected level of mean reversion at time $u$ is equal to:

$$
\mathbb{E}\left(c_{i, u} \mid \mathcal{F}_{s}\right)=\theta_{s}^{\top} \exp \left(Q_{0}(u-s)\right) c_{i}
$$

then expectation of intensities, conditionally to $\mathcal{F}_{s}$ :

$$
\begin{aligned}
& \left(\begin{array}{l}
\mathbb{E}\left(\lambda_{t}^{1} \mid \mathcal{F}_{s}\right) \\
\mathbb{E}\left(\lambda_{t}^{2} \mid \mathcal{F}_{s}\right)
\end{array}\right)=V \int_{s}^{t}\left(\begin{array}{cc}
e^{\gamma_{1}(t-u)} & 0 \\
0 & e^{\gamma_{2}(t-u)}
\end{array}\right) V^{-1}\left(\begin{array}{c}
\kappa_{1} \theta_{s}^{\top} \exp \left(Q_{0}(u-s)\right) c_{1} \\
\kappa_{2} \theta_{s}^{\top} \exp \left(Q_{0}(u-s)\right) c_{2}
\end{array}\right) d u \\
& +V\left(\begin{array}{cc}
e^{\gamma_{1}(t-s)} & 0 \\
0 & e^{\gamma_{2}(t-s)}
\end{array}\right) V^{-1}\left(\begin{array}{c}
\lambda_{s}^{1} \\
\lambda_{s}^{2}
\end{array}\right) \text {. }
\end{aligned}
$$

If we replace $V^{-1}$ by its definition 17 , we obtain that

$$
\begin{aligned}
& V^{-1}\left(\begin{array}{ccc}
\kappa_{1} \theta_{s}^{\top} & \exp \left(Q_{0}(u-s)\right) c_{1} \\
\kappa_{2} \theta_{s}^{\top} & \exp \left(Q_{0}(u-s)\right) c_{2}
\end{array}\right) \\
& \left.=\frac{1}{\Upsilon}\left(\begin{array}{c}
\kappa_{1}\left(\left(\delta_{1,1} \mu_{1}-\kappa_{1}\right)-\gamma_{2}\right) \theta_{s}^{\top} \exp \left(Q_{0}(u-s)\right) c_{1} \\
+\kappa_{2} \delta_{1,2} \mu_{2} \theta_{s}^{\top} \exp \left(Q_{0}(u-s)\right) c_{2} \\
\kappa_{1}\left(\gamma_{1}-\left(\delta_{1,1} \mu_{1}-\kappa_{1}\right)\right) \theta_{s}^{\top} \exp \left(Q_{0}(u-s)\right) c_{1} \\
-\kappa_{2} \delta_{1,2} \mu_{2} \theta_{s}^{\top} \exp \left(Q_{0}(u-s)\right) c_{2}
\end{array}\right)\right) .
\end{aligned}
$$


The integrand in equation (51) becomes then:

$$
\begin{aligned}
& \left(\begin{array}{cc}
e^{\gamma_{1}(t-u)} & 0 \\
0 & e^{\gamma_{2}(t-u)}
\end{array}\right) V^{-1}\left(\begin{array}{ccc}
\kappa_{1} \theta_{s}^{\top} & \exp \left(Q_{0}(u-s)\right) c_{1} \\
\kappa_{2} \theta_{s}^{\top} & \exp \left(Q_{0}(u-s)\right) c_{2}
\end{array}\right)
\end{aligned}
$$

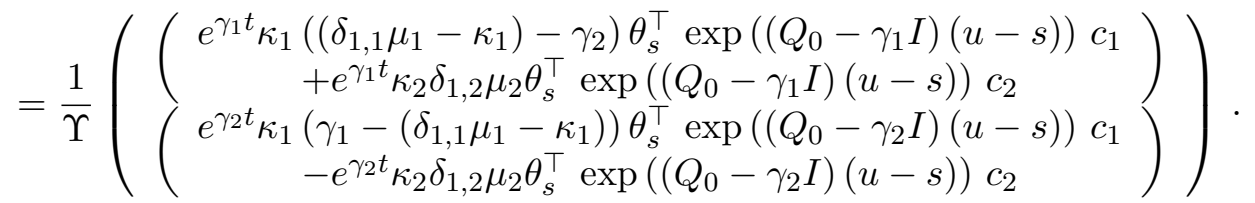

and we can conclude by direct integration that expected value of $\lambda_{t}^{i}$ are given by equation (19). This result also states processes $\lambda_{t}^{1}$ and $\lambda_{t}^{2}$ are Markov given that their $\mathcal{F}_{s}$ expectations only depend on the information available at time $s:\left(\lambda_{s}^{1}, \lambda_{s}^{2}, \theta_{s}^{1}, \theta_{s}^{2}\right)$.

\section{Proof of Corollary 3.3}

To prove this statement, it is sufficient to show that the conditional expectation of these processes with respect to $\mathcal{F}_{s}$ depends exclusively upon the information available at time $s$. Using the Tower property of conditional expectation, the expected number of supply order conditionally to $\mathcal{F}_{s}$ is then equal to the following product:

$$
\mathbb{E}\left(N_{t}^{1} \mid \mathcal{F}_{s}\right)=\mathbb{E}\left(\mathbb{E}\left(N_{t}^{1} \mid \mathcal{F}_{s} \vee \mathcal{H}_{t}\right) \mid \mathcal{F}_{s}\right)
$$

By construction, the compensator of processes $N_{t}^{1}$ is an $\mathcal{H}_{t^{-}}$adapted processes $\int_{0}^{t} \lambda_{u}^{1} d u$ such that the compensated process $M_{t}^{1}=N_{t}^{1}-\int_{0}^{t} \lambda_{u}^{1} d u$ is a martingale. Given that $\mathbb{E}\left(M_{t}^{1} \mid \mathcal{F}_{s} \vee \mathcal{H}_{t}\right)=M_{s}^{1}$, we deduce that $\mathbb{E}\left(N_{t}^{1} \mid \mathcal{F}_{s} \vee \mathcal{H}_{t}\right)=N_{s}^{1}+\int_{s}^{t} \lambda_{u}^{1} d u$. Using the Fubini's theorem, we infer that

$$
\begin{aligned}
\mathbb{E}\left(N_{t}^{1} \mid \mathcal{F}_{s}\right) & =\left(N_{s}^{1}+\mathbb{E}\left(\int_{s}^{t} \lambda_{u}^{1} d u \mid \mathcal{F}_{s}\right)\right) \\
& =N_{s}^{1}+\int_{s}^{t} \mathbb{E}\left(\lambda_{u}^{1} \mid \mathcal{F}_{s}\right) d u .
\end{aligned}
$$

According to proposition 3.2 , the intensity of $N_{t}^{1}$ is a Markov process and $\mathbb{E}\left(\lambda_{u}^{1} \mid \mathcal{F}_{s}\right)$ depends only upon $\lambda_{s}^{1}, \lambda_{s}^{2}$ and $\theta_{s}$ and time $u$. From equation (52), we immediately deduce that $\mathbb{E}\left(N_{t}^{1} \mid \mathcal{F}_{s}\right)$ is exclusively a function of $\left(\lambda_{s}^{1}, \lambda_{s}^{2}, \theta_{s}, N_{s}^{1}\right)$ and then Markov. The same holds for $N_{t}^{2}$. By definition, $L_{t}^{1}$ is a sum of independent random variables:

$$
\begin{aligned}
\mathbb{E}\left(L_{t}^{1} \mid \mathcal{F}_{s}\right) & =\mathbb{E}\left(\sum_{n=1}^{N_{t}^{1}} O_{n}^{1} \mid \mathcal{F}_{s}\right) \\
& =\mu_{1} \mathbb{E}\left(N_{t}^{1} \mid \mathcal{F}_{s}\right)
\end{aligned}
$$

As $\mathbb{E}\left(N_{t}^{1} \mid \mathcal{F}_{s}\right)$ is exclusively a function of $\left(\lambda_{s}^{1}, \lambda_{s}^{2}, \theta_{s}, N_{s}^{1}\right)$, we conclude that $L_{t}^{1}$ is Markov. The same holds for $L_{t}^{2}$.

Proof of proposition 3.4 If we remember the equation (48), we infer that the expectations of 
$c_{j, t} \lambda_{t}^{i}$ for $i, j=1,2$ are solution of ordinary differential equations (ODE):

$$
\begin{aligned}
& \underbrace{\left(\begin{array}{l}
\frac{\partial}{\partial t} \mathbb{E}\left(c_{1, t} \lambda_{t}^{1} \mid \mathcal{F}_{0} \vee \mathcal{G}_{t}\right) \\
\frac{\partial}{\partial t} \mathbb{E}\left(c_{2, t} \lambda_{t}^{1} \mid \mathcal{F}_{0} \vee \mathcal{G}_{t}\right) \\
\frac{\partial}{\partial t} \mathbb{E}\left(c_{1, t} \lambda_{t}^{2} \mid \mathcal{F}_{0} \vee \mathcal{G}_{t}\right) \\
\frac{\partial}{\partial t} \mathbb{E}\left(c_{2, t} \lambda_{t}^{2} \mid \mathcal{F}_{0} \vee \mathcal{G}_{t}\right)
\end{array}\right)}_{:=d E(t)}=\underbrace{\left(\begin{array}{ccc}
\kappa_{1} & 0 & 0 \\
0 & 0 & \kappa_{1} \\
0 & 0 & \kappa_{2} \\
0 & \kappa_{2} & 0
\end{array}\right)}_{:=K} \underbrace{\left(\begin{array}{c}
c_{1, t}^{2} \\
c_{2, t}^{2} \\
c_{1, t} c_{2, t}
\end{array}\right)}_{:=C_{t}^{2}}+ \\
& \underbrace{\left(\begin{array}{cccc}
\delta_{1,1} \mu_{1}-\kappa_{1} & 0 & \delta_{1,2} \mu_{2} & 0 \\
0 & \delta_{1,1} \mu_{1}-\kappa_{1} & 0 & \delta_{1,2} \mu_{2} \\
\delta_{2,1} \mu_{1} & 0 & \delta_{2,2} \mu_{2}-\kappa_{2} & 0 \\
0 & \delta_{2,1} \mu_{1} & 0 & \delta_{2,2} \mu_{2}-\kappa_{2}
\end{array}\right)}_{W F W^{-1}} \underbrace{\left(\begin{array}{l}
\mathbb{E}\left(c_{1, t} \lambda_{t}^{1} \mid \mathcal{F}_{0} \vee \mathcal{G}_{t}\right) \\
\mathbb{E}\left(c_{2, t} \lambda_{t}^{1} \mid \mathcal{F}_{0} \vee \mathcal{G}_{t}\right) \\
\mathbb{E}\left(c_{1, t} \lambda_{t}^{2} \mid \mathcal{F}_{0} \vee \mathcal{G}_{t}\right) \\
\mathbb{E}\left(c_{2, t} \lambda_{t}^{2} \mid \mathcal{F}_{0} \vee \mathcal{G}_{t}\right)
\end{array}\right)}_{E(t)} .
\end{aligned}
$$

We summarize this system of ODE as follows

$$
d E(t)=K C_{t}^{2}+W F W^{-1} E(t) .
$$

If we note $U(t)=W^{-1} E(t)$, we rewrite this last system:

$$
d U(t)=W^{-1} K C_{t}^{2}+F U(t)
$$

that admits the following solution:

$$
U(t)=\int_{0}^{t} \exp (F s) W^{-1} K C_{s}^{2} d s+\exp (F t) U(0)
$$

and we can conclude.

Proof of proposition 3.5. If we remember equation (27), we can develop it as follows

$$
\begin{aligned}
& \exp (F s) W^{-1}\left(\begin{array}{c}
\kappa_{1} c_{1, s}^{2} \\
\kappa_{1} c_{1, s} c_{2, s} \\
\kappa_{2} c_{1, s} c_{2, s} \\
\kappa_{2} c_{2, s}^{2}
\end{array}\right) \\
& =\frac{1}{\Upsilon}\left(\begin{array}{c}
\kappa_{1}\left(\left(\delta_{1,1} \mu_{1}-\kappa_{1}\right)-\gamma_{2}\right)\left(e^{\gamma_{1} s} c_{1, s}^{2}\right)+\delta_{1,2} \mu_{2} \kappa_{2}\left(e^{\gamma_{1} s} c_{1, s} c_{2, s}\right) \\
\kappa_{1}\left(\gamma_{1}-\left(\delta_{1,1} \mu_{1}-\kappa_{1}\right)\right)\left(e^{\gamma_{2} s} c_{1, s}^{2}\right)-\delta_{1,2} \mu_{2} \kappa_{2}\left(e^{\gamma_{2} s} c_{1, s} c_{2, s}\right) \\
\kappa_{1}\left(\gamma_{1}-\left(\delta_{1,1} \mu_{1}-\kappa_{1}\right)\right)\left(e^{\gamma_{2} s} c_{1, s} c_{2, s}\right)-\delta_{1,2} \mu_{2} \kappa_{2}\left(e^{\gamma_{2} s} c_{2, s}^{2}\right)
\end{array}\right),
\end{aligned}
$$

and its expectation is given by

$$
\begin{aligned}
& \mathbb{E}\left(\exp (F s) W^{-1}\left(\begin{array}{c}
\kappa_{1} c_{1, s}^{2} \\
\kappa_{1} c_{1, s} c_{2, s} \\
\kappa_{2} c_{1, s} c_{2, s} \\
\kappa_{2} c_{2, s}^{2}
\end{array}\right)\right) \\
& =\frac{1}{\Upsilon}\left(\begin{array}{l}
\kappa_{1}\left(\left(\delta_{1,1} \mu_{1}-\kappa_{1}\right)-\gamma_{2}\right)\left(\theta_{0} e^{\left(Q_{0}+\gamma_{1} I\right) s} \bar{c}_{1}^{2}\right)+\delta_{1,2} \mu_{2} \kappa_{2}\left(\theta_{0} e^{\left(Q_{0}+\gamma_{1} I\right) s} \bar{c}_{1,2}\right) \\
\kappa_{1}\left(\gamma_{1}-\left(\delta_{1,1} \mu_{1}-\kappa_{1}\right)\right)\left(\theta_{0} e^{\left(Q_{0}+\gamma_{2} I\right) s} \bar{c}_{1}^{2}\right)-\delta_{1,2} \mu_{2} \kappa_{2}\left(\theta_{0} e^{\left(Q_{0}+\gamma_{2} I\right) s} \bar{c}_{1,2}\right) \\
\kappa_{1}\left(\left(\delta_{1,1} \mu_{1}-\kappa_{1}\right)-\gamma_{2}\right)\left(\theta_{0} e^{\left(Q_{0}+\gamma_{1} I\right) s} \bar{c}_{1,2}\right)+\delta_{1,2} \mu_{2} \kappa_{2}\left(\theta_{0} e^{\left(Q_{0}+\gamma_{1} I\right) s} \bar{c}_{2}^{2}\right) \\
\kappa_{1}\left(\gamma_{1}-\left(\delta_{1,1} \mu_{1}-\kappa_{1}\right)\right)\left(\theta_{0} e^{\left(Q_{0}+\gamma_{2} I\right) s} \bar{c}_{1,2}\right)-\delta_{1,2} \mu_{2} \kappa_{2}\left(\theta_{0} e^{\left(Q_{0}+\gamma_{2} I\right) s} \bar{c}_{2}^{2}\right)
\end{array}\right) .
\end{aligned}
$$


Integrating this last equation allows us to conclude.

Proof of proposition 3.6. If we remember the expression 24 of the infinitesimal generator, we have

$$
\begin{aligned}
\mathcal{A}\left(\left(\lambda_{t}^{1}\right)^{2}\right)= & 2 \kappa_{1}\left(c_{1, t}-\lambda_{t}^{1}\right) \lambda_{t}^{1}+\lambda_{t}^{1} \int_{-\infty}^{+\infty}\left(\lambda_{t}^{1}+\delta_{1,1} z\right)^{2}-\left(\lambda_{t}^{1}\right)^{2} \nu_{1}(d z) \\
& +\lambda_{t}^{2} \int_{-\infty}^{+\infty}\left(\lambda_{t}^{1}+\delta_{1,2} z\right)^{2}-\left(\lambda_{t}^{1}\right)^{2} \nu_{2}(d z), \\
\mathcal{A}\left(\left(\lambda_{t}^{2}\right)^{2}\right)= & 2 \kappa_{2}\left(c_{2, t}-\lambda_{t}^{2}\right) \lambda_{t}^{2}+\lambda_{t}^{1} \int_{-\infty}^{+\infty}\left(\lambda_{t}^{2}+\delta_{2,1} z\right)^{2}-\left(\lambda_{t}^{2}\right)^{2} \nu_{1}(d z) \\
& +\lambda_{t}^{2} \int_{-\infty}^{+\infty}\left(\lambda_{t}^{2}+\delta_{2,2} z\right)^{2}-\left(\lambda_{t}^{2}\right)^{2} \nu_{2}(d z), \\
\mathcal{A}\left(\lambda_{t}^{1} \lambda_{t}^{2}\right)= & \kappa_{1}\left(c_{1, t}-\lambda_{t}^{1}\right) \lambda_{t}^{2}+\kappa_{2}\left(c_{2, t}-\lambda_{t}^{2}\right) \lambda_{t}^{1} \\
& +\lambda_{t}^{1} \int_{-\infty}^{+\infty}\left(\lambda_{t}^{1}+\delta_{1,1} z\right)\left(\lambda_{t}^{2}+\delta_{2,1} z\right)-\lambda_{t}^{1} \lambda_{t}^{2} \nu_{1}(d z) \\
& +\lambda_{t}^{2} \int_{-\infty}^{+\infty}\left(\lambda_{t}^{1}+\delta_{1,2} z\right)\left(\lambda_{t}^{2}+\delta_{2,2} z\right)-\lambda_{t}^{1} \lambda_{t}^{2} \nu_{2}(d z) .
\end{aligned}
$$

And given that $\frac{\partial}{\partial t} g=\mathbb{E}\left(\mathcal{A} g \mid \mathcal{F}_{0}\right)$, we can conclude.

Proof of proposition 3.9. Let us assume that $\theta_{t}=e_{i}$. If we denote by $g\left(\lambda_{t}^{1}, J_{t}^{1}, \lambda_{t}^{2}, J_{t}^{2}, \theta_{t}\right)=$ $\mathbb{E}\left(\omega^{N_{s}^{k}} \mid \mathcal{F}_{t}\right), g$ is solution of the following Itô's equation for semi martingale :

$$
\begin{aligned}
0= & g_{t}+\kappa_{1}\left(c_{1, t}-\lambda_{t}^{1}\right) g_{\lambda^{1}}+\kappa_{2}\left(c_{2, t}-\lambda_{t}^{2}\right) g_{\lambda^{2}} \\
& +\lambda_{t}^{1} \int_{-\infty}^{+\infty} g\left(\lambda_{t}^{1}+\delta_{1,1} z, J_{t}^{1}+(z, 1)^{\top}, \lambda_{t}^{2}+\delta_{2,1} z, J_{t}^{2}, e_{i}\right)-g\left(\lambda_{t}^{1}, J_{t}^{1}, \lambda_{t}^{2}, J_{t}^{2}, e_{i}\right) d \nu_{1}(z) \\
& +\lambda_{t}^{2} \int_{-\infty}^{+\infty} g\left(\lambda_{t}^{1}+\delta_{1,2} z, J_{t}^{1}, \lambda_{t}^{2}+\delta_{2,2} z, J_{t}^{2}+(z, 1)^{\top}, e_{i}\right)-g\left(\lambda_{t}^{1}, J_{t}^{1}, \lambda_{t}^{2}, J_{t}^{2}, e_{i}\right) d \nu_{2}(z) \\
& +\sum_{j \neq i}^{N} q_{i, j}\left(g\left(\lambda_{t}^{1}, J_{t}^{1}, \lambda_{t}^{2}, J_{t}^{2}, e_{j}\right)-g\left(\lambda_{t}^{1}, J_{t}^{1}, \lambda_{t}^{2}, J_{t}^{2}, e_{i}\right)\right) .
\end{aligned}
$$

Next, we assume that $g$ is an exponential affine function of $\lambda_{t}^{1}, \lambda_{t}^{2}$ and $N_{t}^{i}$ :

$$
g=\exp \left(A\left(t, s, e_{j}\right)+B_{1}(t, s) \lambda_{t}^{1}+B_{2}(t, s) \lambda_{t}^{2}+C(t, s) N_{t}^{k}\right)
$$

where $A\left(t, s, e_{i}\right)$ for $i=1$ to $N, B_{1}(t, s), B_{2}(t, s)$ and $C(t, s)$ are time dependent functions. The partial derivatives of $g$ are then given by:

$$
\begin{gathered}
g_{t}=\left(\frac{\partial}{\partial t} A\left(t, s, e_{j}\right)+\frac{\partial}{\partial t} B_{1}(t, s) \lambda_{t}^{1}+\frac{\partial}{\partial t} B_{2}(t, s) \lambda_{t}^{2}+\frac{\partial}{\partial t} C(t, s) N_{t}^{k}\right) g \\
g_{\lambda^{1}}=B_{1}(t, s) g \quad \text { and } \quad g_{\lambda^{2}}=B_{2}(t, s) g
\end{gathered}
$$


And the integrands in equation (53) are rewritten with the notations $A:=A\left(t, s, e_{i}\right), B_{1}:=B_{1}(t, s)$, $B_{2}:=B_{2}(t, s)$ and $C:=C(t, s)$ as follows:

$$
\begin{aligned}
& \int_{-\infty}^{+\infty} g\left(\lambda_{t}^{1}+\delta_{1,1} z, J_{t}^{1}+(z, 1)^{\top}, \lambda_{t}^{2}+\delta_{2,1} z, J_{t}^{2}, e_{i}\right)-g\left(\lambda_{t}^{1}, J_{t}^{1}, \lambda_{t}^{2}, J_{t}^{2}, e_{i}\right) d \nu_{1}(z) \\
& \quad=g\left[e^{1_{k=1} C} \psi_{1}\left(B_{1} \delta_{1,1}+B_{2} \delta_{2,1}\right)-1\right] \\
& \quad \int_{-\infty}^{+\infty} g\left(\lambda_{t}^{1}+\delta_{1,2} z, J_{t}^{1}, \lambda_{t}^{2}+\delta_{2,2} z, J_{t}^{2}+(z, 1)^{\top}, e_{j}\right)-g\left(\lambda_{t}^{1}, J_{t}^{1}, \lambda_{t}^{2}, J_{t}^{2}, e_{i}\right) d \nu_{2}(z) \\
& \quad=g\left[e^{1_{k=2} C} \psi_{2}\left(B_{1} \delta_{1,2}+B_{2} \delta_{2,2}\right)-1\right] .
\end{aligned}
$$

As the sum of instantaneous probabilities is null, $q_{i i}=-\sum_{i \neq j}^{N} q_{i, j}$, we have that

$$
\sum_{j \neq i}^{N} q_{i, j}\left(g\left(\lambda_{t}^{1}, J_{t}^{1}, \lambda_{t}^{2}, J_{t}^{2}, e_{j}\right)-g\left(\lambda_{t}^{1}, J_{t}^{1}, \lambda_{t}^{2}, J_{t}^{2}, e_{i}\right)\right)=\sum_{j=1}^{N} q_{i, j} g\left(\lambda_{t}^{1}, J_{t}^{1}, \lambda_{t}^{2}, J_{t}^{2}, e_{j}\right) .
$$

Then the equation 53 becomes:

$$
\begin{aligned}
0= & \left(\frac{\partial}{\partial t} A+\frac{\partial}{\partial t} B_{1} \lambda_{t}^{1}+\frac{\partial}{\partial t} B_{2} \lambda_{t}^{2}+\frac{\partial}{\partial t} C N_{t}^{i}\right) e^{A\left(t, s, e_{i}\right)} \\
& +\kappa_{1}\left(c_{1, t}-\lambda_{t}^{1}\right) B_{1} e^{A\left(t, s, e_{i}\right)}+\kappa_{2}\left(c_{2, t}-\lambda_{t}^{2}\right) B_{2} e^{A\left(t, s, e_{i}\right)} \\
& +\lambda_{t}^{1}\left(e^{1_{k=1} C} \psi_{1}\left(B_{1} \delta_{1,1}+B_{2} \delta_{2,1}\right)-1\right) e^{A\left(t, s, e_{i}\right)} \\
& +\lambda_{t}^{2}\left(e^{1_{k=2} C} \psi_{2}\left(B_{1} \delta_{1,2}+B_{2} \delta_{2,2}\right)-1\right) e^{A\left(t, s, e_{i}\right)} \\
& +\sum_{j=1}^{N} q_{i, j} g\left(\lambda_{t}^{1}, J_{t}^{1}, \lambda_{t}^{2}, J_{t}^{2}, e_{j}\right)
\end{aligned}
$$

from which we guess that $C(t, s)=\ln \omega$. Regrouping terms allows to infer that

$$
\begin{aligned}
& 0=\frac{\partial}{\partial t} A e^{A\left(t, s, e_{i}\right)}+\kappa_{1} c_{1, t} B_{1} e^{A\left(t, s, e_{i}\right)}+\kappa_{2} c_{2, t} B_{2} e^{A\left(t, s, e_{i}\right)}+\sum_{j=1}^{N} q_{i, j} e^{A\left(t, s, e_{j}\right)} \\
& +\lambda_{t}^{1}\left(\frac{\partial}{\partial t} B_{1}-\kappa_{1} B_{1}+\left[1_{k=1} \omega \psi_{1}\left(B_{1} \delta_{1,1}+B_{2} \delta_{2,1}\right)-1\right]\right) e^{A\left(t, s, e_{i}\right)} \\
& +\lambda_{t}^{2}\left(\frac{\partial}{\partial t} B_{2}-\kappa_{2} B_{2}+\left[1_{k=2} \omega \psi_{2}\left(B_{1} \delta_{1,2}+B_{2} \delta_{2,2}\right)-1\right]\right) e^{A\left(t, s, e_{i}\right)}
\end{aligned}
$$

Given that $\lambda_{t}^{1}$ and $\lambda_{t}^{2}$ are random quantities, this equation is satisfied if and only if

$$
\begin{aligned}
\frac{\partial}{\partial t} B_{1} & =\kappa_{1} B_{1}-\left[1_{k=1} \omega \psi_{1}\left(B_{1} \delta_{1,1}+B_{2} \delta_{2,1}\right)-1\right] \\
\frac{\partial}{\partial t} B_{2} & =\kappa_{2} B_{2}-\left[1_{k=2} \omega \psi_{2}\left(B_{1} \delta_{1,2}+B_{2} \delta_{2,2}\right)-1\right] \\
\left(\frac{\partial}{\partial t} A\right) e^{A\left(t, s, e_{i}\right)} & =-\kappa_{1} c_{1, t} B_{1} e^{A\left(t, s, e_{i}\right)}-\kappa_{2} c_{2, t} B_{2} e^{A\left(t, s, e_{i}\right)}-\sum_{j=1}^{N} q_{i, j} e^{A\left(t, s, e_{j}\right)} .
\end{aligned}
$$


If we define $\tilde{A}\left(t, s, e_{i}\right)=e^{A\left(t, s, e_{i}\right)}$, the last equations can finally be put in matrix form as:

$$
\frac{\partial \tilde{A}(t)}{\partial t}+\left(\operatorname{diag}\left(\kappa_{1} c_{1, t} B_{1}+\kappa_{2} c_{2, t} B_{2}\right)+Q_{0}\right) \tilde{A}(t)=0 .
$$

Proof of proposition 3.11. From previous results, we know that $B_{k}(t, s)$ is solution of the following ODE

$$
\frac{\partial}{\partial t} B_{k}=\kappa_{k} B_{k}+(-1)^{k} \omega_{1} \alpha_{k} \mu_{k}-\left[\psi_{k}\left(B_{1} \delta_{1, k}+B_{2} \delta_{2, k}+C_{k}\right)-1\right], \quad k=1,2
$$

with terminal condition $B_{k}(s, s)=\omega_{k+1}$. If we set $B_{k}(t, s)=D_{k}(s-t)$ and $\tau=s-t$. Then

$$
\frac{\partial B_{k}}{\partial t}=\frac{\partial D_{k}}{\partial \tau} \frac{\partial \tau}{\partial t}=-\frac{\partial D_{k}}{\partial \tau}
$$

Thus we obtain

$$
\begin{aligned}
\frac{\partial D_{k}}{\partial \tau} & =-\kappa_{k} B_{k}(\tau)-(-1)^{k} \omega_{1} \alpha_{k} \mu_{k}+\left[\psi_{k}\left(D_{1}(\tau) \delta_{1, k}+D_{2}(\tau) \delta_{2, k}+C_{k}\right)-1\right] \\
& =-\kappa_{k} D_{k}(\tau)+\psi_{k}\left(D_{1}(\tau) \delta_{1, k}+D_{2}(\tau) \delta_{2, k}+C_{k}\right)-\left[(-1)^{k} \omega_{1} \alpha_{k} \mu_{k}+1\right] \\
& =-\kappa_{k} D_{k}(\tau)+\psi_{k}\left(D_{1}(\tau) \delta_{1, k}+D_{2}(\tau) \delta_{2, k}+C_{k}\right)-\beta_{k}\left(\omega_{1}\right)
\end{aligned}
$$

The left hand side is then denoted $h_{k}\left(D_{1}, D_{2}\right)$. Due to the convexity of $\psi_{k}$ there is only one point $\left(u_{1}^{*}, u_{2}^{*}\right)$ such that $h_{k}(u)=0$ for $k=1,2$. These equations are indeed equivalent to

$$
\psi_{k}\left(u_{1} \delta_{1, k}+u_{2} \delta_{2, k}+C_{k}\right)=\beta_{k}\left(\omega_{1}\right)+\kappa_{k} u_{k} .
$$

We rewrite the equations 55 as follows,

$$
\frac{d D_{k}}{-\kappa_{k} D_{k}+\psi_{k}\left(D_{1} \delta_{1, k}+D_{2} \delta_{2, k}+C_{k}\right)-\beta_{k}\left(\omega_{1}\right)}=d \tau
$$

As $D_{k}(0)=\omega_{k+1}$ for $k \in\{1,2\}$ by direct integration, we have that

$$
\begin{aligned}
& \int_{\omega_{2}}^{D_{1}} \frac{d u_{1}}{-\kappa_{1} u_{1}+\psi_{1}\left(u_{1} \delta_{1,1}+D_{2} \delta_{2,1}+C_{1}\right)-\beta_{1}\left(\omega_{1}\right)}=\tau \\
& \int_{\omega_{3}}^{D_{2}} \frac{d u_{2}}{-\kappa_{2} u_{2}+\psi_{2}\left(D_{1} \delta_{1,2}+u_{2} \delta_{2,2}+C_{2}\right)-\beta_{2}\left(\omega_{1}\right)}=\tau
\end{aligned}
$$

with $D_{k} \in\left[\omega k+1, u_{k}^{*}\right)$ or $D_{k} \in\left[u_{k}^{*}, \omega k+1\right)$.

We can remark that if $\left(D_{1}, D_{2}\right)=\left(u_{1}^{*}, u_{2}^{*}\right)$ then $\tau=+\infty$ as the numerator converges to zero. If we define the functions $F_{\omega_{1}}^{1}(x, y)$ and $F_{\omega_{1}}^{2}(x, y)$ from $\mathbb{R}^{2}$ to $\mathbb{R}^{+}$by equations $(36), D_{1}$ and $D_{2}$ are such that $F_{\omega_{1}}^{k}\left(D_{1}, D_{2}\right)=\tau$. If $\left(F_{\omega_{1}}^{1}\right)^{-1}(\tau \mid y)$ and $\left(F_{\omega_{1}}^{2}\right)^{-1}(\tau \mid x)$ are respectively the inverse functions of $F_{\omega_{1}}^{1}(., y)$ and $F_{\omega_{1}}^{2}(x,$.$) , then D_{1}$ and $D_{2}$ satisfy the following system

$$
\begin{aligned}
& D_{1}=\left(F_{\omega_{1}}^{1}\right)^{-1}\left(\tau \mid D_{2}\right) \\
& D_{2}=\left(F_{\omega_{1}}^{2}\right)^{-1}\left(\tau \mid D_{1}\right)
\end{aligned}
$$

or $B_{1}(t, s)=\left(F_{\omega_{1}}^{1}\right)^{-1}\left(s-t \mid B_{2}(t, s)\right)$ and $B_{2}(t, s)=\left(F_{\omega_{1}}^{2}\right)^{-1}\left(s-t \mid B_{1}(t, s)\right)$. 


\section{Acknowledgment}

We thank for its support the Chair "Data Analytics and Models for insurance" of BNP Paribas Cardiff, hosted by ISFA (Universite Claude Bernard, Lyon France). We also thank the two anonymous referees and the editor, Ulrich Horst, for their recommandations.

\section{References}

[1] Ait-Sahalia, Y., Cacho-Diaz, J., Laeven, R.J.A. 2015. Modeling financial contagion using mutually exciting jump processes. J. of Fin. Econ. 117(3), 586-606.

[2] Al-Anaswah N., Wilfing B. 2011. Identification of speculative bubbles using state-space models with Markov-switching. Journal of Banking \& Finance, 35(5), 1073-1086.

[3] Bacry E., Delattre S., Hoffmann M., Muzy J.F., 2013 (a). Modelling microstructure noise with mutually exciting point processes. Quantitative Finance 13(1), 65-77.

[4] Bacry E., Delattre S., Hoffmann M., Muzy J.F., 2013 (b). Scaling limits for Hawkes processes and application to financial statistics. Stochastic Processes and Applications, 123 (7), 2475-2499.

[5] Bacry E. Muzy J.F. 2014. Hawkes model for price and trades high-frequency dynamics. Quantitative Finance, 14 (7), 1147-1166.

[6] Bacry E., Mastromatteo I., Muzy J.F. 2015. Hawkes Processes in Finance. Market Microstructure and Liquidity 1(1), pp 1-59.

[7] Bacry E. Muzy J.F. 2016. Second order statistics characterization of Hawkes processes and non-parametric estimation. IEEE Transactions on Information Theory, 62 (4), 2184-2202.

[8] Bormetti G., Calcagnile L.M., Treccani M., Corsi F., Marmi S., Lillo F. 2015. Modelling systemic price cojumps with Hawkes factor models. Quantitative Finance 15 (7), 1137-1156.

[9] Bouchaud J.P. 2010, Price impact, in Encyclopedia of Quantitative Finance, R. Cont, ed., John Wiley \& Sons Ltd.

[10] Bouchaud J.P., Farmer J.D., Lillo F. 2009. How markets slowly diggest changes in supply and demand, in Handbook of Financial Markets, Elsevier.

[11] Bowsher, C. G. 2002. Modelling security markets in continuous time: Intensity based, multivariate point process models. Economics Discussion Paper No. 2002- W22, Nuffield College, Oxford.

[12] Branger N., Kraft H., Meinerding C., 2014. Partial information about contagion risk, selfexciting processes and portfolio optimization. Journal of Economic Dynamics and Control 39, $18-36$.

[13] Chavez-Demoulin, V., McGill J.A. 2012. High-frequency financial data modeling using Hawkes processes. Journal of Banking and Finance, 36, 3415-3426.

[14] Cont R., Kukanov A., Stoikov S., 2013. The price impact of order book events. Journal of financial econometrics, 12(1), 47-88. 
[15] Da Fonseca J., Zaatour R. 2014. Hawkes process: Fast calibration, application to trade clustering, and diffusive limit. Journal of futures markets, 34(6), 548-579.

[16] Filimonov V., Sornette D., 2015. Apparent criticality and calibration issues in the hawkes selfexcited point process model: application to high-frequency financial data. Quantitative Finance 15(8), 1293-1314.

[17] Gatumel M., Ielpo F. 2014. The Number of Regimes Across Asset Returns: Identification and Economic Value. International Journal of Theoretical and Applied Finance, 17(06), 25 pages.

[18] Guidolin M., Timmermann A. 2005. Economic Implications of bull and bear regimes in UK stock and bond returns. The Economic Journal, 115, 11-143.

[19] Guidolin M., Timmermann A. 2008. International Asset Allocation under Regime Switching, Skew, and Kurtosis Preferences. Review of Financial Studies. 21 (2), 889-935.

[20] Hainaut D. 2016 a. A model for interest rates with clustering effects. Quantitative Finance, 16 (8), 1203-1218.

[21] Hainaut D. 2016 b. A bivariate Hawkes process for interest rate modeling. Economic Modelling 57, 180-196.

[22] Hainaut D. 2017. Clustered Lévy processes and their financial applications. Journal of Computational and Applied Mathematics 319, 117-140.

[23] Hainaut D. MacGilchrist R. 2012. Strategic asset allocation with switching dependence. Annals of Finance, 8(1), 75-96.

[24] Hardiman S.J. , Bouchaud. J.P. 2014. Branching ratio approximation for the self-exciting Hawkes process. Physical review E. 90(6), 628071-628076.

[25] Hautsch, N. 2004. Modelling Irregularly Spaced Financial Data. Springer, Berlin.

[26] Hawkes, A., 1971(a). Point sprectra of some mutually exciting point processes. Journal of the Royal Statistical Society Series B, 33, 438-443.

[27] Hawkes, A., 1971(b). Spectra of some self-exciting and mutually exciting point processes. Biometrika, 58, 83-90.

[28] Hawkes A. and Oakes D., 1974. A cluster representation of a self-exciting process. Journal of Applied Probability, 11, 493-503.

[29] Horst U., Paulsen M. 2017. A Law of Large Numbers for Limit Order Books. Mathematics of operations research, Online : https://doi.org/10.1287/moor.2017.0848

[30] Jaisson T., Rosenbaum M. 2015. Limit theorems for nearly unstable hawkes processes. The annals of applied probability 25(2), pp 600-631.

[31] Kelly F., Yudovina E., 2017. A Markov Model of a Limit Order Book: Thresholds, Recurrence, and Trading Strategies. Mathematics of operations research, Online : https://doi.org/10.1287/moor.2017.0857

[32] Kyle A.S. 1985. Continuous auction and insider trading, Econometrica 53, p. 1315-1335. 
[33] Large, J. 2005. Measuring the resiliency of an electronic limit order book. Working Paper, All Souls College, University of Oxford.

[34] Lee K., Seo B.K. 2017. Modeling microstructure price dynamics with symmetric Hawkes and diffusion model using ultra-high-frequency stock data. Journal of Economic Dynamics and Control, 79, 154-183.

[35] Protter P.E. 2004. Stochastic integration and differential equations. Springer-Verlag Berlin Hedeilberg.

[36] Wang T., Bebbington M., Harte D. 2012. Markov-modulated Hawkes process with stepwise decay. Ann. Inst. Stat. Math. 64, 521-544. 